\title{
Sub-nm Pt Clusters on Defective NiFe LDH Nanosheets as Trifunctional Electrocatalysts for Water Splitting and Rechargeable \\ Hybrid Sodium-air Battery
}

Xueqing Yu, ${ }^{\mathrm{a}}$ Junpo Guo, ${ }^{\mathrm{a}} \mathrm{Bo} \mathrm{Li},{ }^{\mathrm{a}}$ Jincheng Xu, ${ }^{\mathrm{a}}$ Peng Gao, ${ }^{* \mathrm{~b}} \mathrm{Kwan}$ San Hui, ${ }^{\mathrm{c}} \mathrm{Kw}$ w Nam Hui, ${ }^{* \mathrm{a}}$ and Huaiyu Shao*a

a Joint Key Laboratory of the Ministry of Education, Institute of Applied Physics and Materials Engineering, University of Macau, Avenida da Universidade, Taipa, Macau SAR, China. Email: hshao@um.edu.mo, bizhui@um.edu.mo

b School of Chemistry and Molecular Bioscience, University of Wollongong, NSW 2500, Australia. Email:pg177@uowmail.edu.au

${ }^{c}$ School of Engineering, Faculty of Science, University of East Anglia, Norwich, NR4 7TJ, United Kingdom. 


\section{Experimental Section}

\section{Characterization of Materials}

The as-prepared samples were characterized by scanning electron microscopy (SEM, Zeiss Sigma) and transmission electron microscopy (TEM, Talos F200S, 200kV). The crystal structures were analyzed by a powder X-ray diffractometer (XRD, Rigaku Smartlab, CuK $\alpha 1$ ). The Raman spectroscopy was conducted on Horiba LABHRev-UV. The X-ray photoelectron spectroscopy (XPS) was collected on Thermo Scientific ESCALAB Xi ${ }^{+}$XPS Microprobe.

\section{Electrochemical measurements}

EIS data were acquired within the operating frequencies of $100 \mathrm{kHz}$ to $1 \mathrm{~Hz}$ with the sweeping potential of $50 \mathrm{mV}$ from the equilibrium potential. The electrochemically active surface area (ECSA) of as-synthesized samples was measured at the non-Faradaic region range 1.17-1.27 V vs. RHE in 1 $\mathrm{M} \mathrm{KOH}$, and the scan rates were 10, 25, 50, 100 and $200 \mathrm{mV} \mathrm{s}^{-1}$, respectively. The double-layer capacitance $\left(\mathrm{C}_{\mathrm{dl}}\right)$ was calculated by plotting the $\Delta j\left(j_{a}-j_{c}\right)$ at $1.22 \mathrm{~V}$ against the scan rate, $j_{a}$ and $j_{c}$ represented the anodic and cathodic current density, respectively, and the linear slope is equivalent to $2 \mathrm{C}_{\mathrm{dl}}$.

The turn over frequency (TOF, $\mathrm{s}^{-1}$ ) of as-prepared catalysts for HER and OER were calculated with the following equation: ${ }^{1}$

$$
T O F=\frac{j}{n N F}
$$

where $J$ (in A) was the measured current during the linear sweep measurement. $n$ represented total electron number consumed to form one hydrogen $(n=2)$ or oxygen molecule $(n=4)$. N (in mol) was the number of active sites and calculated according to the equation:

$$
\mathrm{N}=\mathrm{Q} / 2 \mathrm{~F}=(\mathrm{It}) / 2 \mathrm{~F}=(\mathrm{IV} / \mu) / 2 \mathrm{~F}=\mathrm{S} /(2 \mathrm{~F} \mu)
$$

$\mathrm{S}$ was the integrated effective area in $\mathrm{CV}$ recorded from 1.17 to $1.27 \mathrm{~V}$ with a scan rate $(\mu)$ of $10 \mathrm{mV}$ $\mathrm{s}^{-1}$. F displayed the Faraday constant $\left(96,485 \mathrm{C} \mathrm{mol}^{-1}\right)$.

The benchmark catalyst was prepared by dispersing $5 \mathrm{mg}$ ground catalyst into $0.95 \mathrm{~mL}$ ethanol and $0.05 \mathrm{~mL}$ Nafion solution ( $5 \mathrm{wt} \%$, Sigma-Aldrich) mixed solution, then ultrasonication for at least $30 \mathrm{~min}$ to form homogeneous catalyst ink. The mass loading of $20 \% \mathrm{Pt} / \mathrm{C}$ (Hesen), $20 \% \mathrm{Ir} / \mathrm{C}$ (Premetek) and $\mathrm{RuO}_{2}$ (Macklin) on $\mathrm{Ni}$ foam was $0.1 \mathrm{mg} \mathrm{cm}{ }^{-2}$.

\section{Theoretical Calculations}

Computational simulation-based electrocatalytic HER in alkaline solution mechanism: ${ }^{2}$

$$
\begin{aligned}
*+\mathrm{H}_{2} \mathrm{O}+\mathrm{e}^{-} \rightarrow{ }^{*} \mathrm{H}+\mathrm{OH}^{-} & \text {Volmer step } \\
{ }^{*} \mathrm{H}+\mathrm{H}_{2} \mathrm{O}+\mathrm{e}^{-} \rightarrow \mathrm{H}_{2}+\mathrm{OH}^{-} & \text {Heyrovsky step }
\end{aligned}
$$




$$
{ }^{*} \mathrm{H}+{ }^{*} \mathrm{H} \rightarrow \mathrm{H}_{2} \quad \text { Tafel step }
$$

The electrocatalytic OER in alkaline solution mechanism: ${ }^{2}$

$$
\begin{gathered}
*+\mathrm{H}_{2} \mathrm{O} \rightarrow * \mathrm{OH}+\mathrm{H}^{+}+\mathrm{e}^{-} \\
* \mathrm{OH} \rightarrow * \mathrm{O}+\mathrm{H}^{+}+\mathrm{e}^{-} \\
* \mathrm{O}+\mathrm{H}_{2} \mathrm{O} \rightarrow * \mathrm{OOH}+\mathrm{H}^{+}+\mathrm{e}^{-} \\
* \mathrm{OOH} \rightarrow *+\mathrm{O}_{2}+\mathrm{H}^{+}+\mathrm{e}^{-}
\end{gathered}
$$

Here, * represents the active sites in the catalyst. 

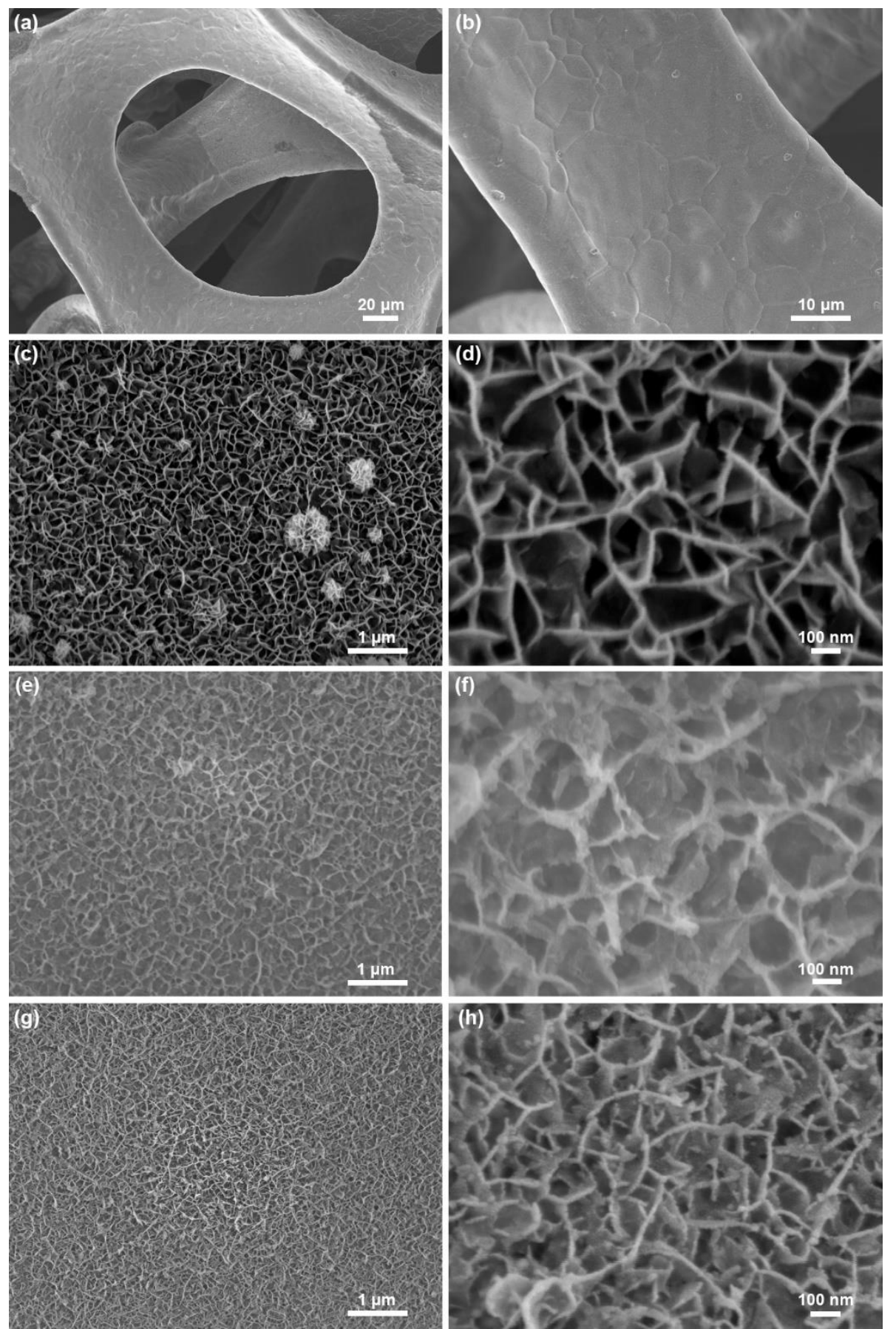

Figure S1. SEM images of $(a, b)$ bare Ni foam, (c, d) NiFe LDHs, (e,f) Pt/NiFe LDHs, and (g, h) $\mathrm{Pt} / \mathrm{NiZnFe} \mathrm{LDHs}$ at different magnifications. 

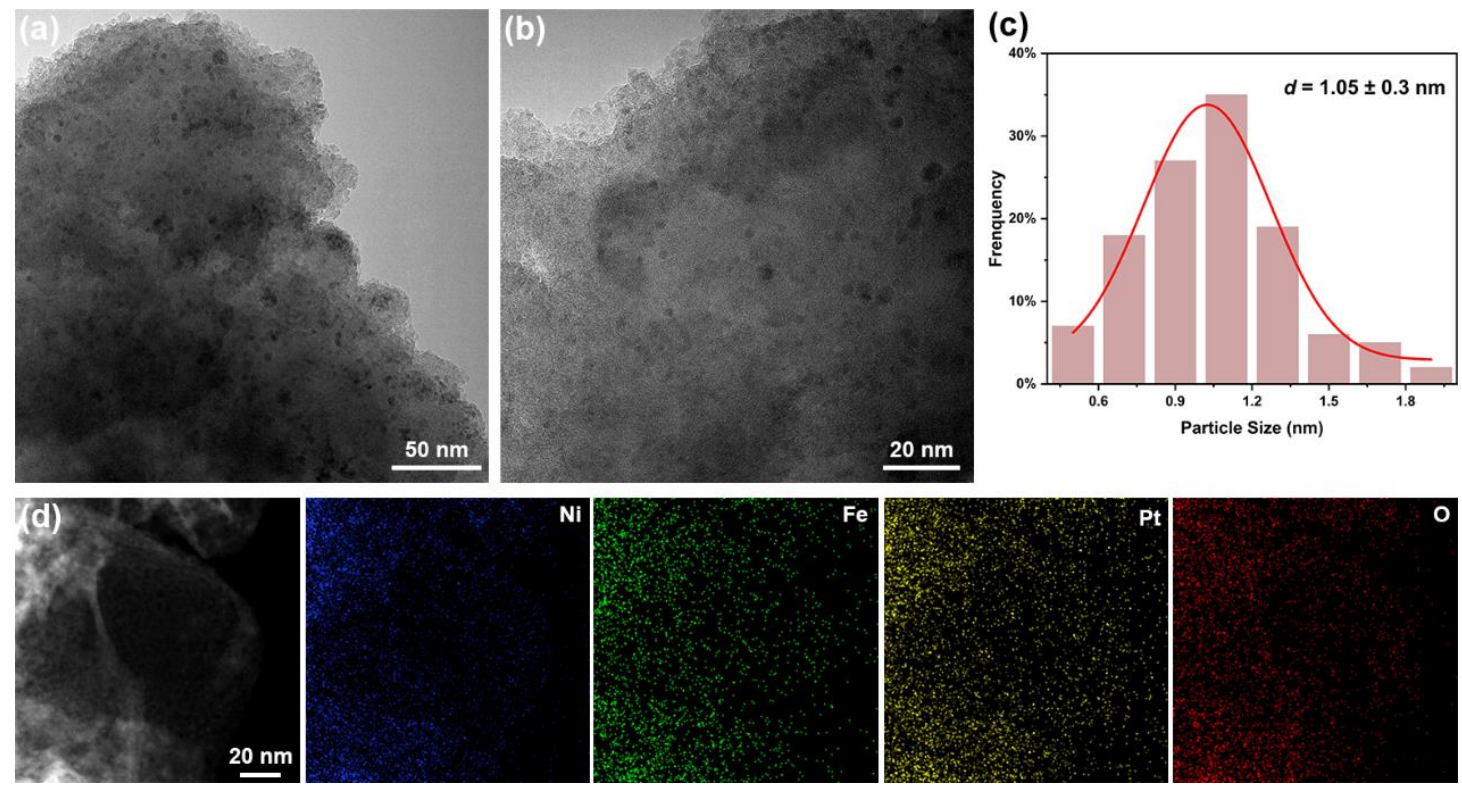

Figure S2. (a, b) TEM images and (c) particle size distribution of deposited Pt of Pt/NiZnFe LDHs. (d) STEM-EDS elemental mapping images of $\mathrm{Pt} / \mathrm{Ni} \mathrm{F}_{\mathrm{F}} \mathrm{LDHs}$.
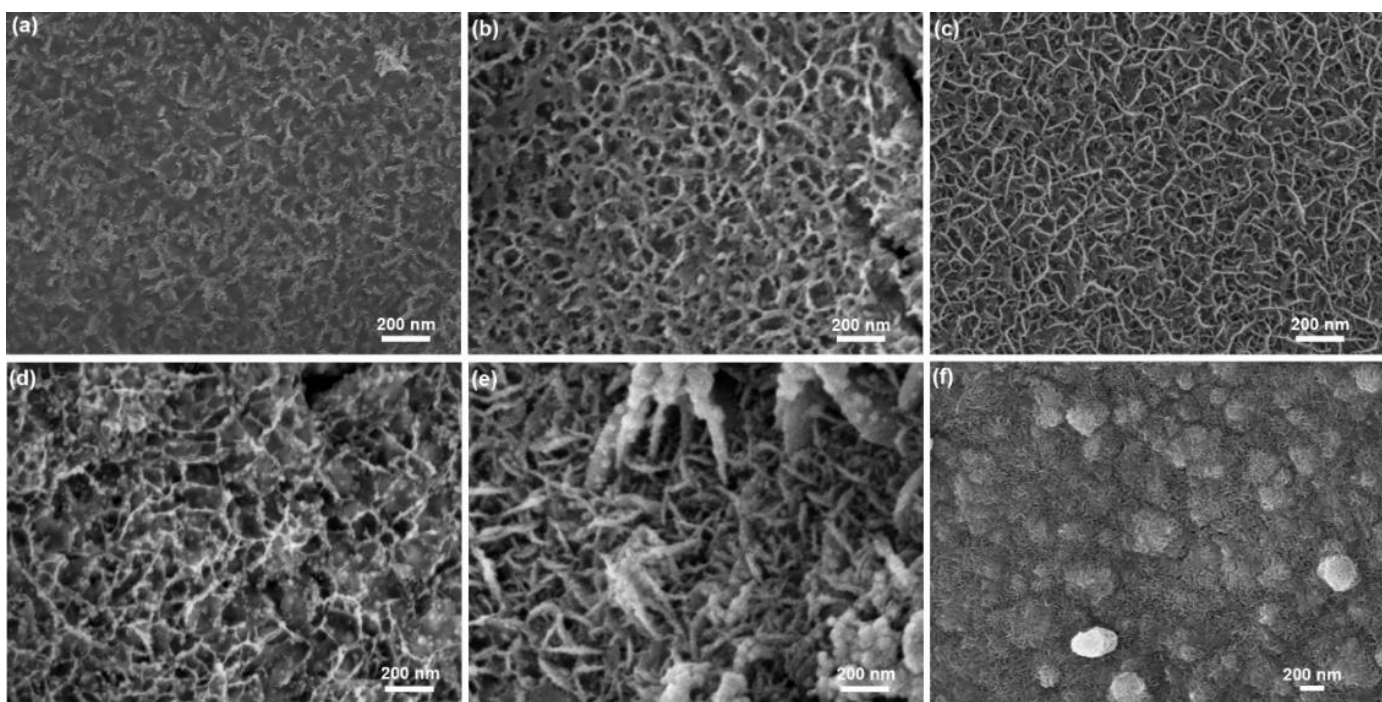

Figure S3. SEM images of $\mathrm{Pt} / \mathrm{Ni}_{\mathrm{x}} \mathrm{Fe} \mathrm{LDHs}$ obtained at room temperature with different deposition time of (a) $100 \mathrm{~s}$, (b) $200 \mathrm{~s}$, (c) $300 \mathrm{~s}$, (d) $400 \mathrm{~s}$, (e) $500 \mathrm{~s}$, and (f) at $0{ }^{\circ} \mathrm{C}$ with deposition time of $300 \mathrm{~s}$. 


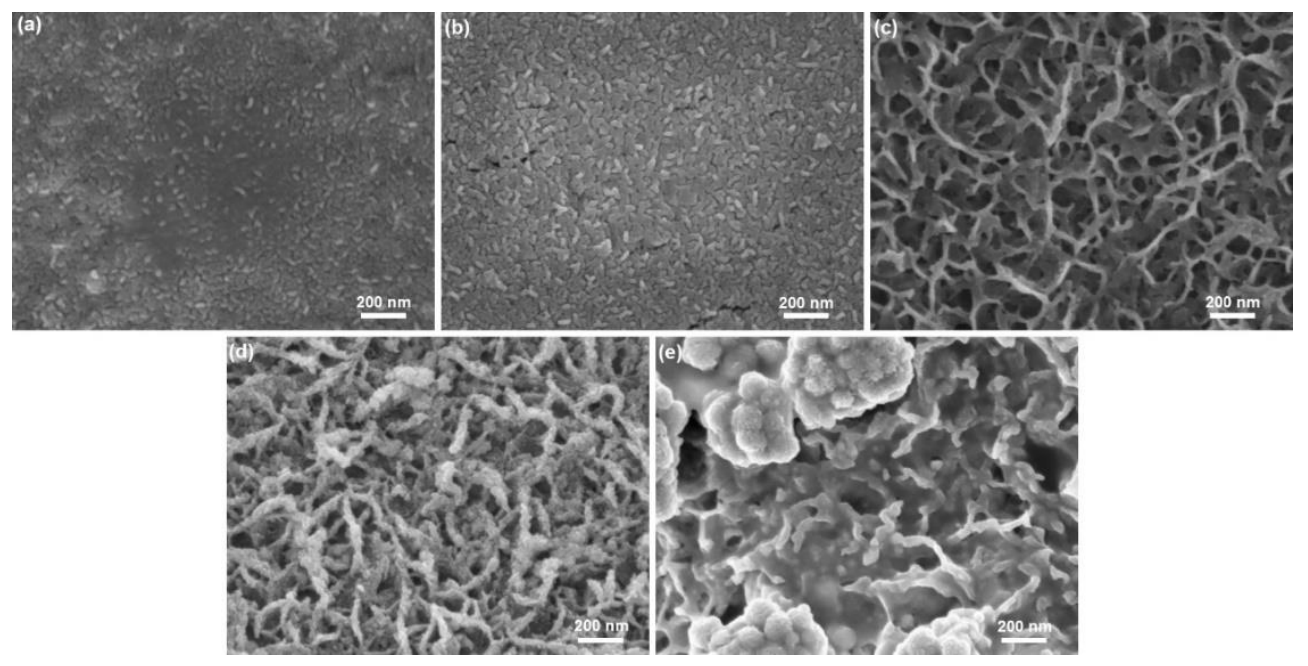

Figure S4. SEM images of $\mathrm{Pt} / \mathrm{Ni}_{\mathrm{x}} \mathrm{Fe}$ LDHs obtained at room temperture with different deposition potential of (a) $-0.1 \mathrm{~V}$, (b) $-0.5 \mathrm{~V}$, (c) $-1.0 \mathrm{~V}$, (d) $-1.5 \mathrm{~V}$, and (e) $-2.0 \mathrm{~V}$.

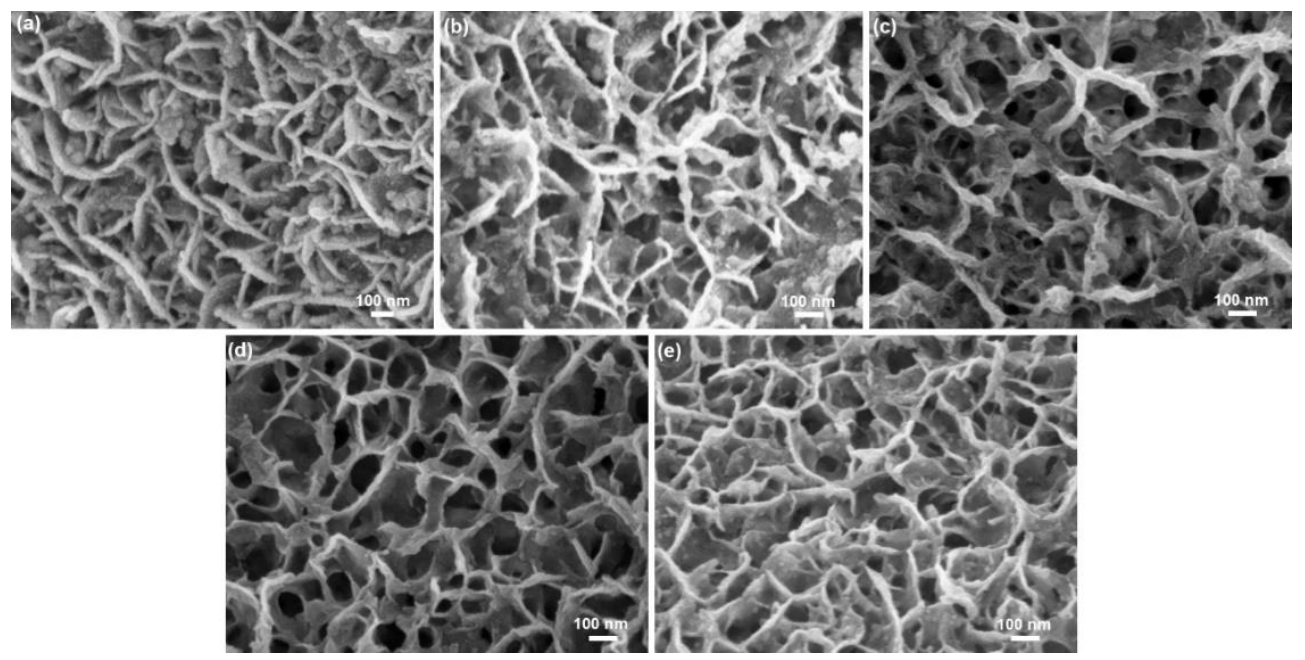

Figure S5. SEM images of $\mathrm{Pt} / \mathrm{Ni}_{x} \mathrm{Fe}$ LDHs obtained at room temperature with different basic-treated time of (a) $0 \mathrm{~h},(\mathrm{~b}) 0.5 \mathrm{~h}$, (c) $1.0 \mathrm{~h}$, (d) $2.5 \mathrm{~h}$, and (e) $5.0 \mathrm{~h}$.
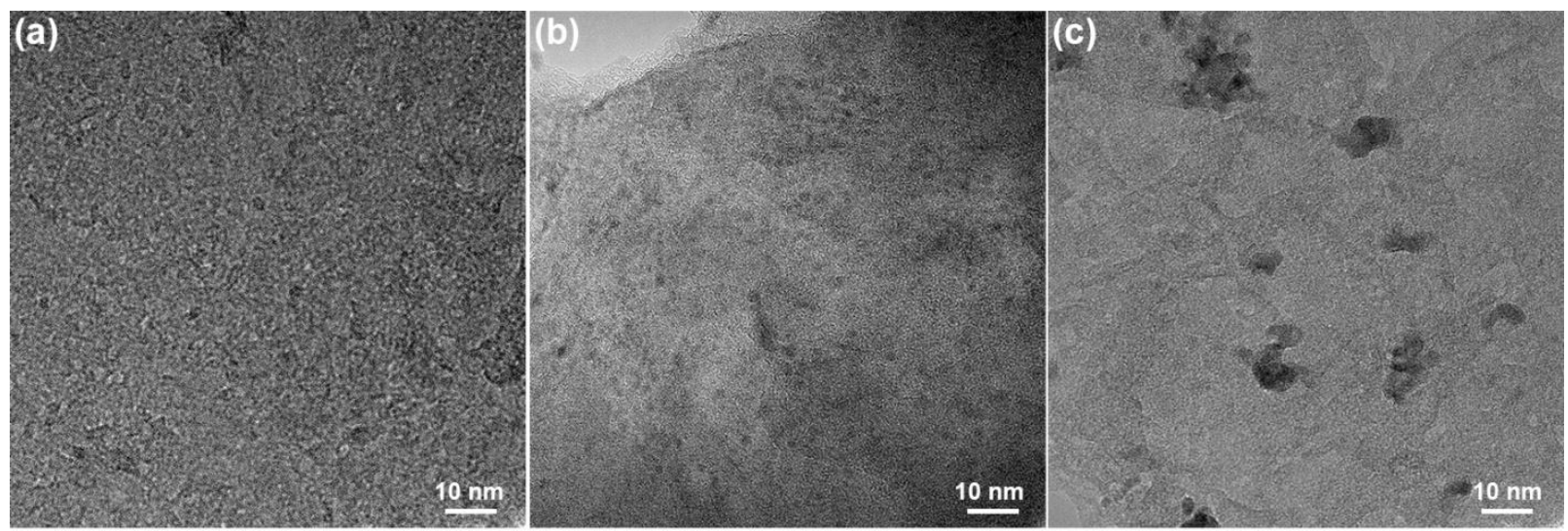

Figure S6. TEM images of Pt/NixFe LDHs with different concentration of Pt precursors: (a) 1.0, (b) 2.0, and (c) $3.0 \mathrm{mM}$. 

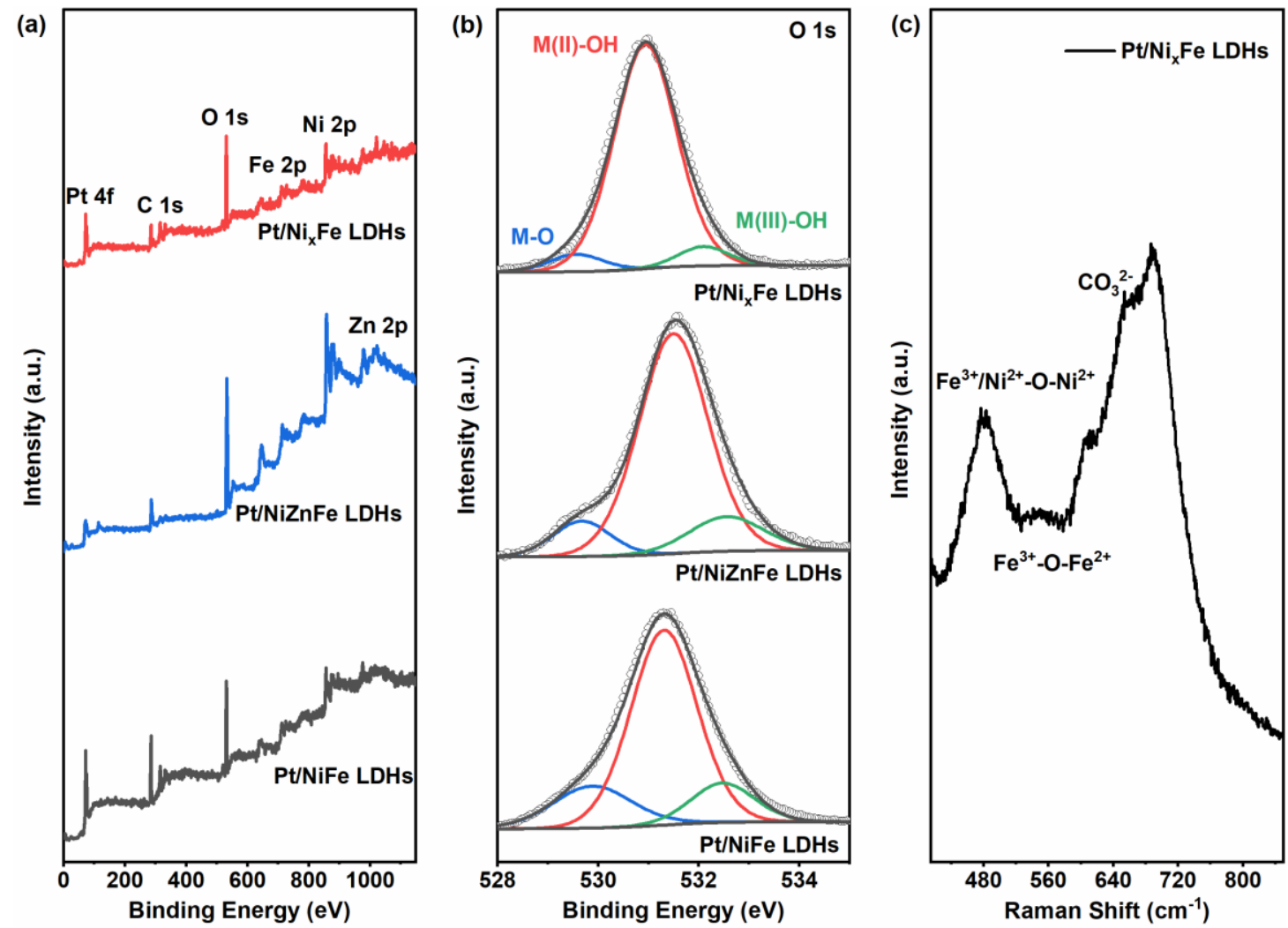

Figure S7. (a) XPS spectra and deconvoluted of (b) $\mathrm{O}$ 1s in $\mathrm{Pt} / \mathrm{Ni}_{\mathrm{x}} \mathrm{Fe} \mathrm{LDHs}, \mathrm{Pt} / \mathrm{NiZnFe} \mathrm{LDHs}$ and $\mathrm{Pt} / \mathrm{NiFe}$ LDHs. (c) Raman spectra were obtained at $633 \mathrm{~nm}$ of $\mathrm{Pt} / \mathrm{Ni} \mathrm{x}_{\mathrm{x}} \mathrm{Fe}$ LDHs.
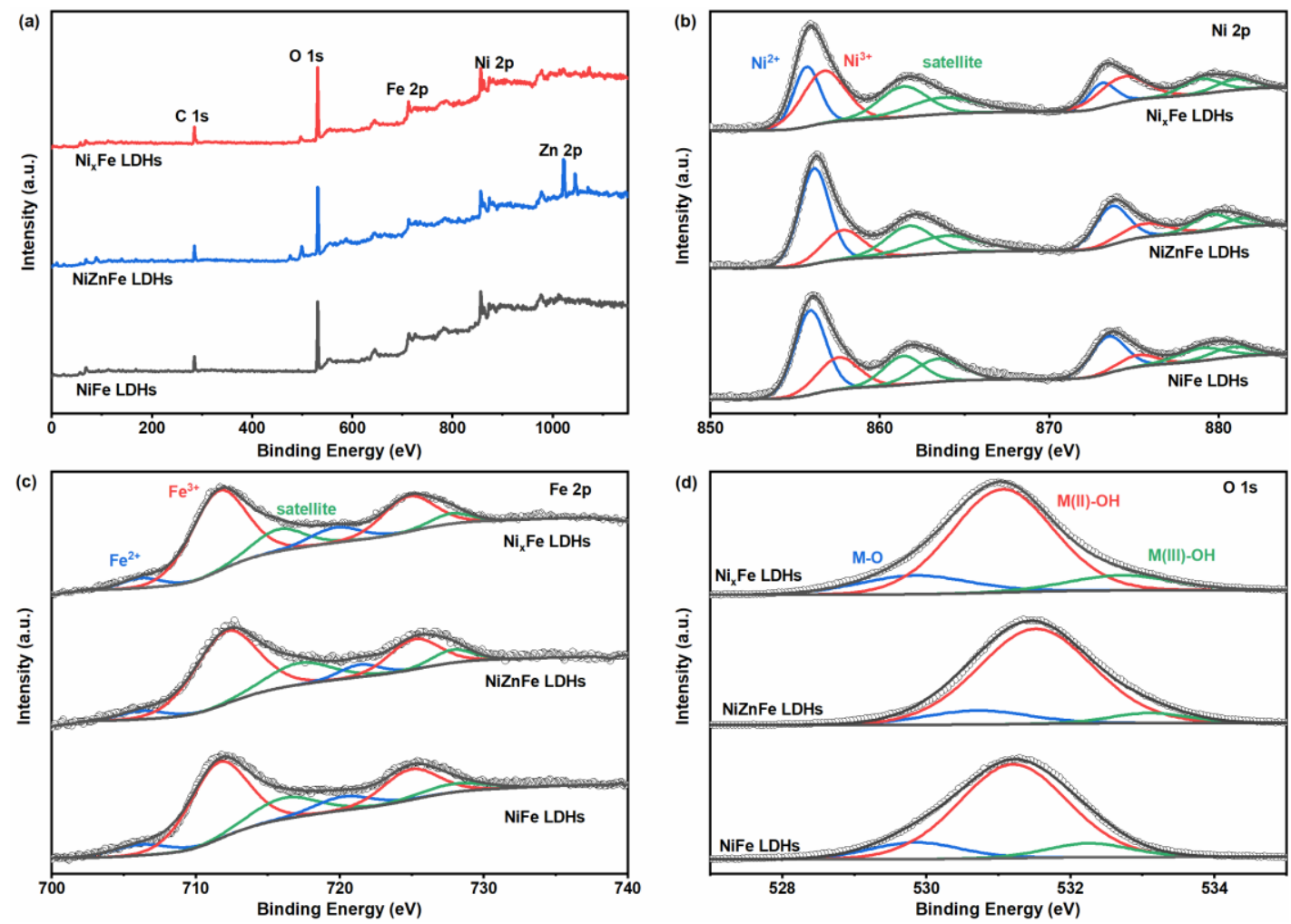

Figure S8. (a) XPS spectra and deconvoluted of (b) Ni 2p, (c) Fe 2p, and (d) O 1s in NixFe LDHs, NiZnFe LDHs, and NiFe LDHs. 

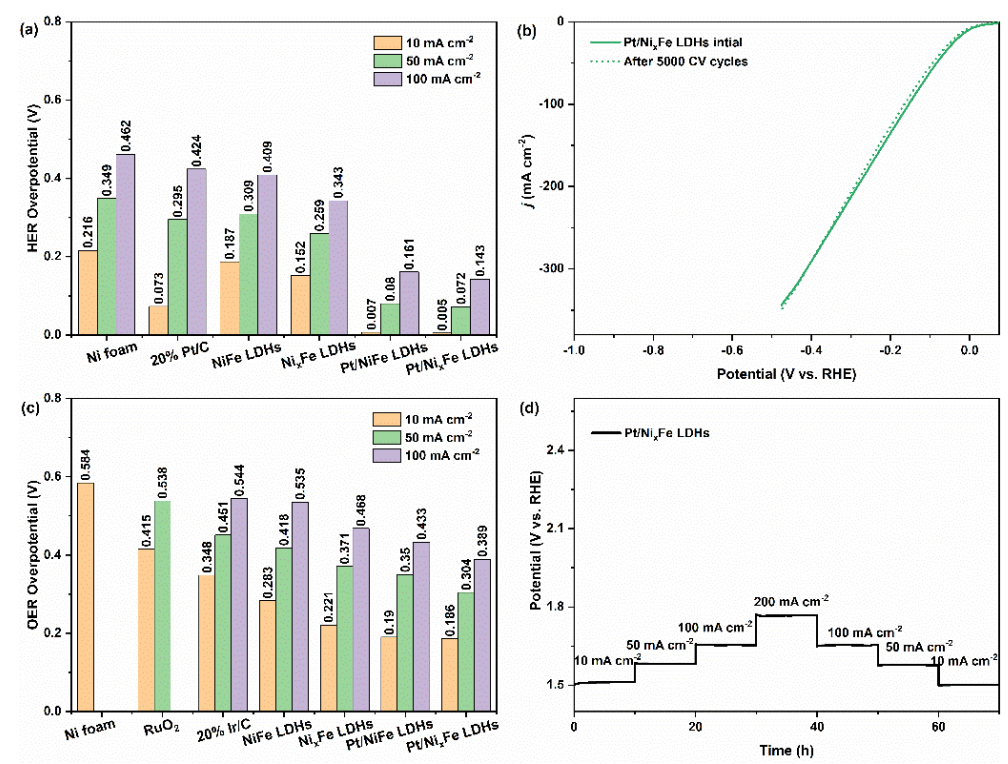

Figure S9. (a) HER overpotentials of $\mathrm{Ni}$ foam, $20 \% \mathrm{Pt} / \mathrm{C}$, NiFe LDHs, $\mathrm{Ni}_{x} \mathrm{Fe}$ LDHs, Pt/NiFe LDHs, and $\mathrm{Pt} / \mathrm{Ni}{ }_{x} \mathrm{Fe}$ LDHs measured at different current densities. (b) HER LSVs of Pt/Ni $\mathrm{x}_{\mathrm{x}} \mathrm{Fe} \mathrm{LDHs}$ catalyst initially and after 5000 cycles. (c) OER overpotentials of $\mathrm{Ni}$ foam, $\mathrm{RuO}_{2}, 20 \% \mathrm{It} / \mathrm{C}, \mathrm{NiFe} \mathrm{LDHs}$, $\mathrm{Ni}_{\mathrm{x}} \mathrm{Fe}$ LDHs, Pt/NiFe LDHs, and Pt/Ni ${ }_{x} \mathrm{Fe}$ LDHs measured at different current densities. (d) OER multi-step chronopotentiometric process of $\mathrm{Pt} / \mathrm{Ni} \mathrm{x}_{\mathrm{Fe}} \mathrm{LDHs}$ catalyst obtained with different current densities.
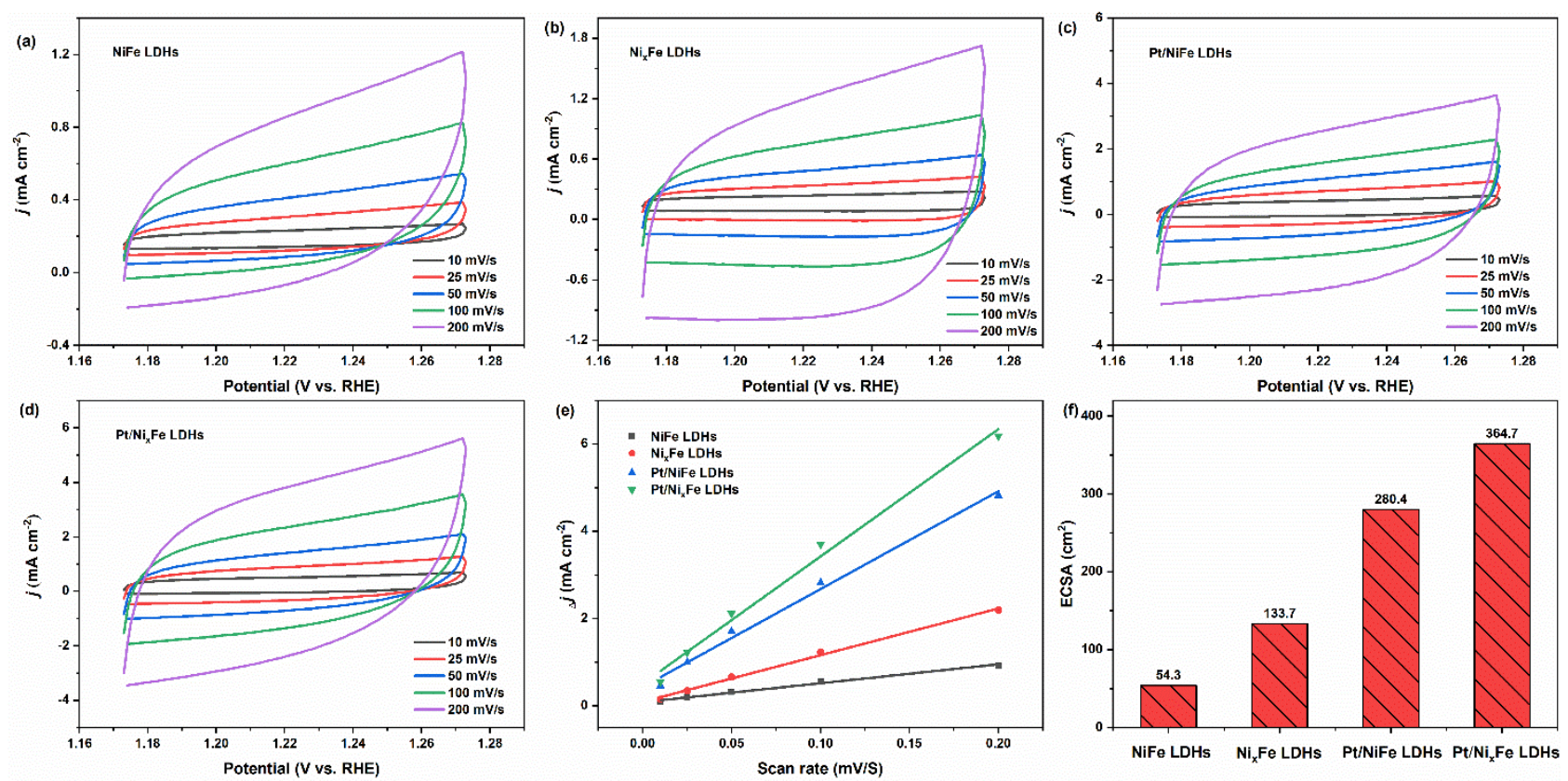

Figure S10. Cyclic voltammograms obtained with (a) NiFe LDHs, (b) $\mathrm{Ni}_{x} \mathrm{Fe}$ LDHs, (c) Pt/NiFe LDHs, and (d) Pt/NixFe LDHs in the capacitance current range (1.173-1.273 V) at scan rates of 10, 25, 50, 100 and $200 \mathrm{mV} \mathrm{s}^{-1}$, respectively. (d) The plot of the difference between double-layer charging currents $(j a-j c)$ vs. scan rate. (e) ESCA of various catalysts. 

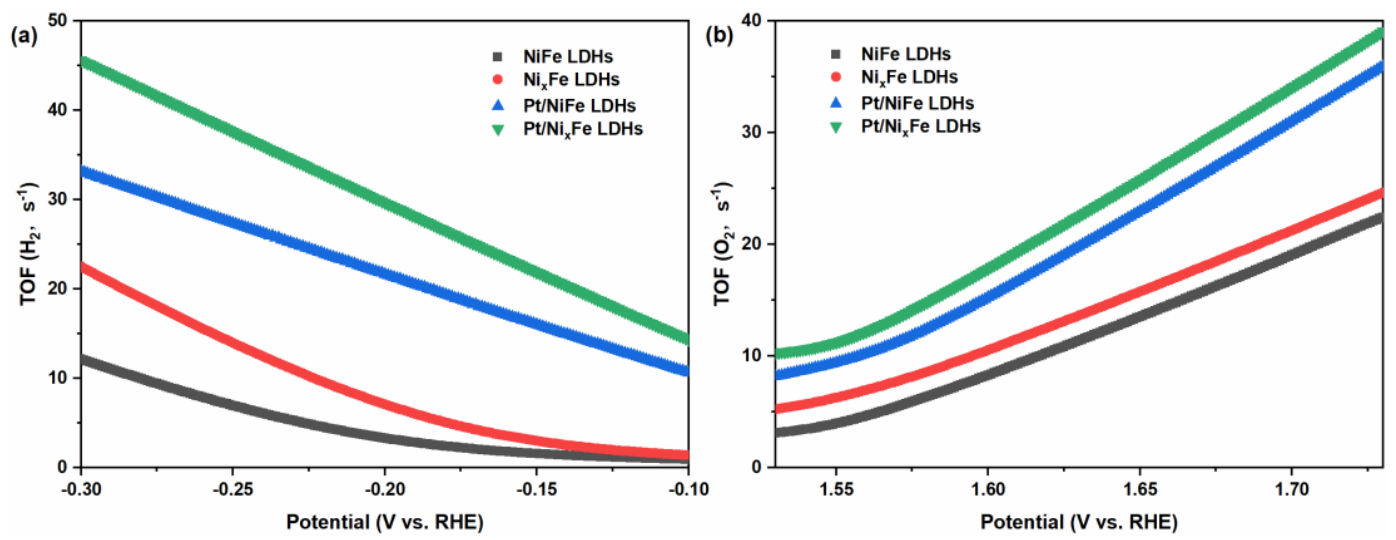

Figure S11. TOF values of NiFe LDHs, $\mathrm{Ni}_{x} \mathrm{Fe}$ LDHs, $\mathrm{Pt} / \mathrm{NiFe} \mathrm{LDHs}$, and $\mathrm{Pt} / \mathrm{Ni} \mathrm{F}_{\mathrm{F}} \mathrm{LDHs}$ electrodes for (a) HER and (b) OER in $1 \mathrm{M} \mathrm{KOH}$ solution, respectively.
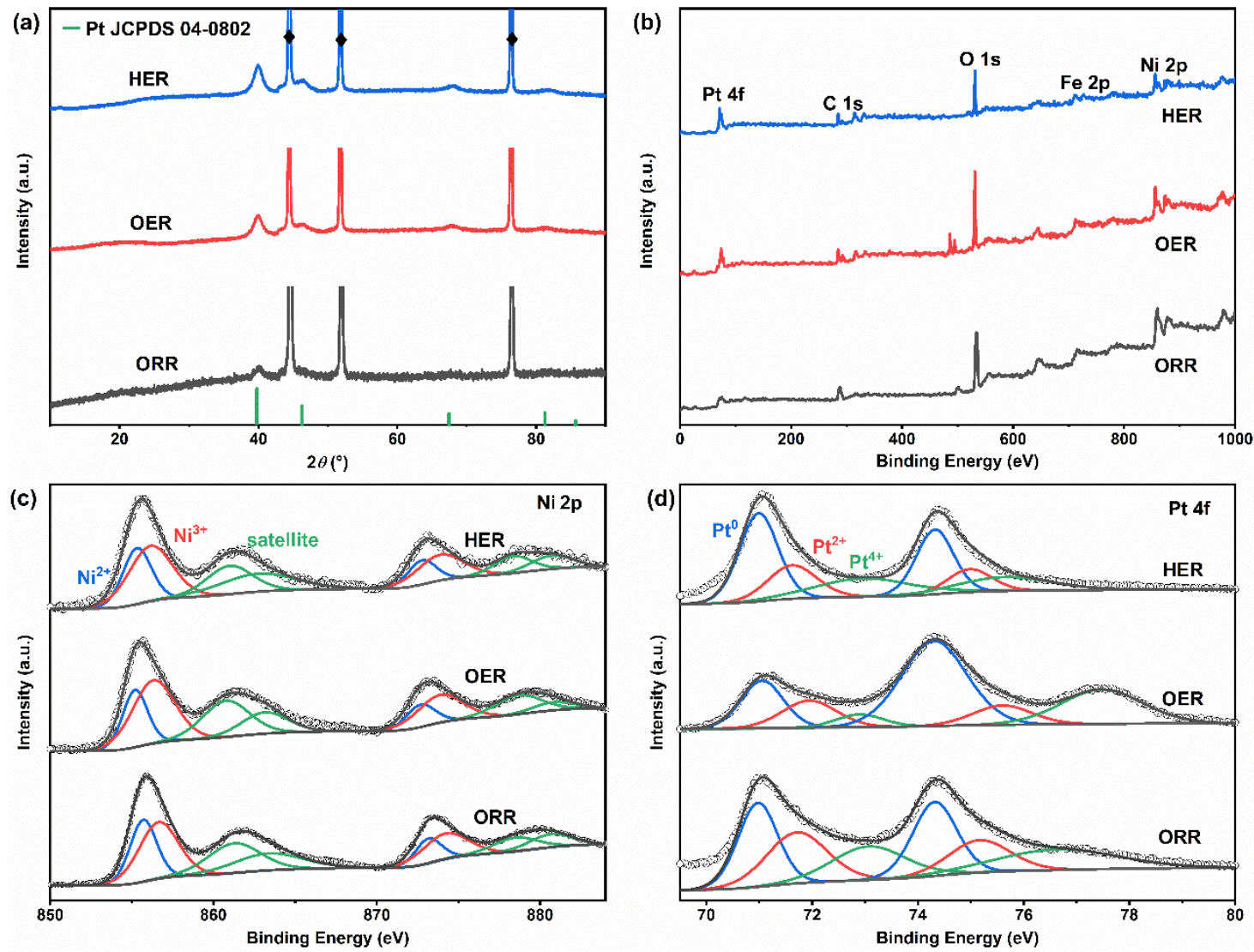

Figure S12. (a) XRD pattern, (b) XPS spectra and deconvoluted of (c) Ni $2 \mathrm{p}$ and (d) Pt $4 \mathrm{f}$ of Pt/Nix $\mathrm{Fe}$ LDHs after long term HER, OER, and ORR stability tests. (The peaks marked " " stand for the Ni substrate.) 

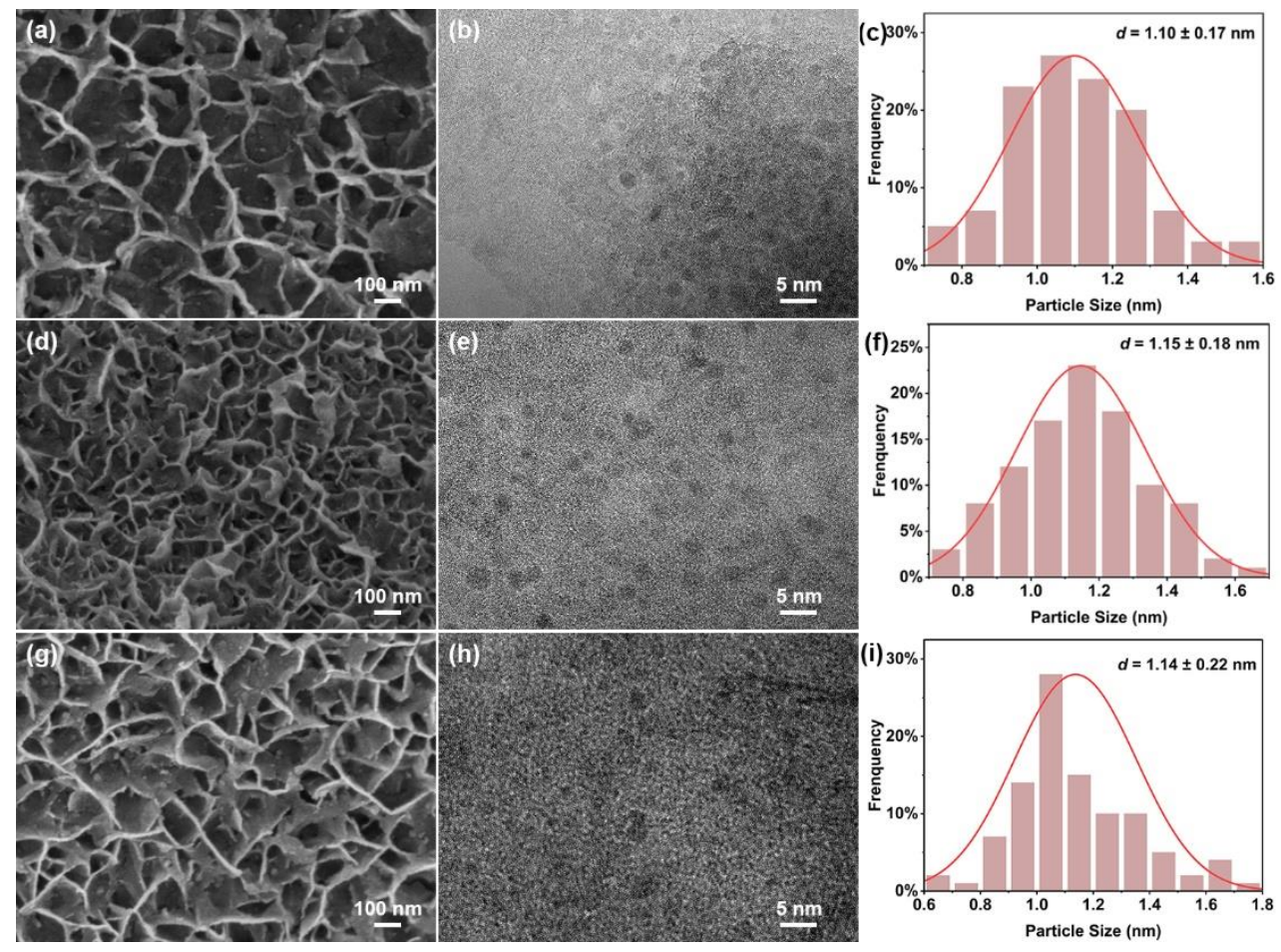

Figure S13. SEM, TEM images, and $\mathrm{Pt} \mathrm{NCs}$ size distribution histogram of $\mathrm{Pt} / \mathrm{Ni}_{\mathrm{x}} \mathrm{Fe} \mathrm{LDHs}$ after long term (a-c) HER, (d-f) OER, and (g-i) ORR stability tests.
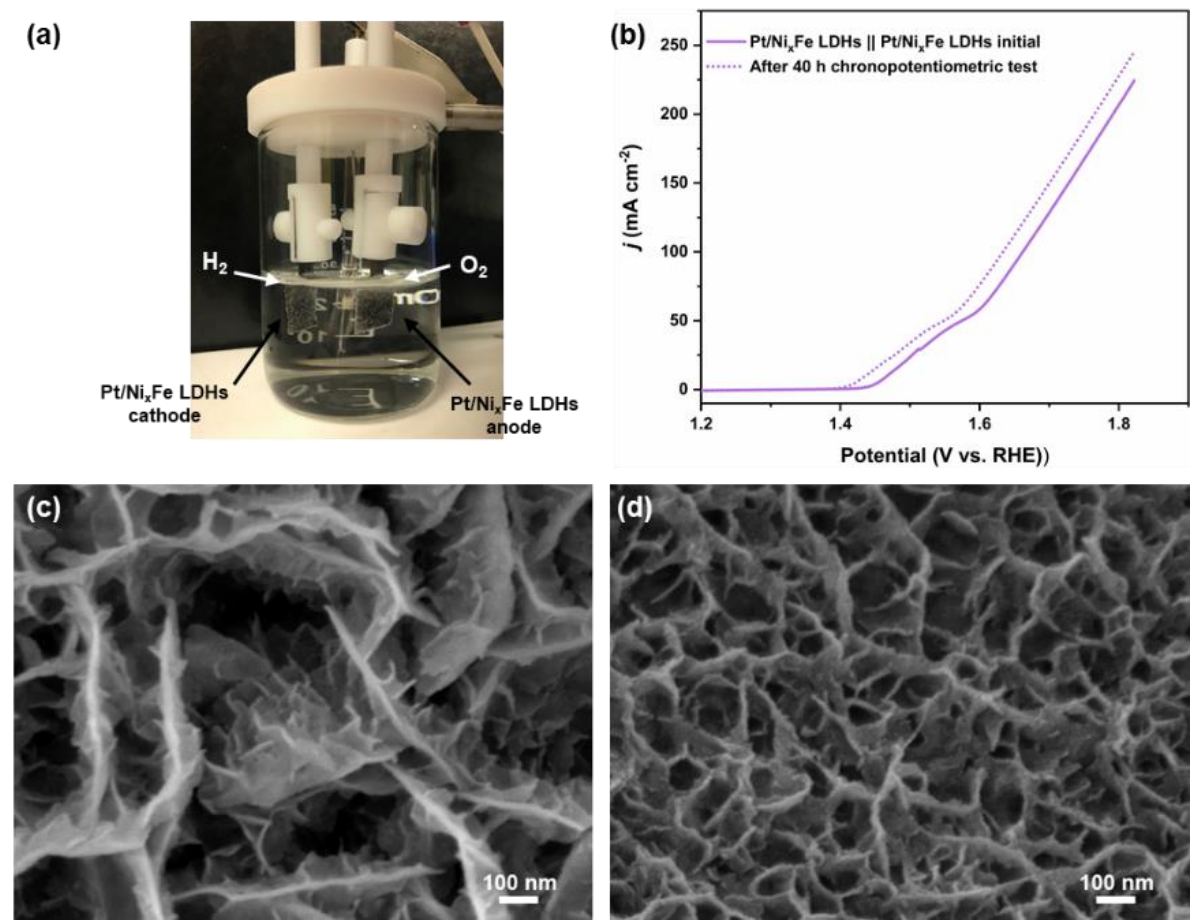

Figure S14. (a) Optical photograph of the overall water splitting device. (b) Polarization curves were obtained on the working Pt/Ni ${ }_{x} F e$ LDHs electrodes initially and after $40 \mathrm{~h}$ chronopotentiometric test. SEM images of (c) $\mathrm{Pt} / \mathrm{Ni}_{\mathrm{x}} \mathrm{Fe}$ LDHs cathode and (d) $\mathrm{Pt} / \mathrm{Ni}_{\mathrm{x}} \mathrm{Fe}$ LDHs anode after stability test. 

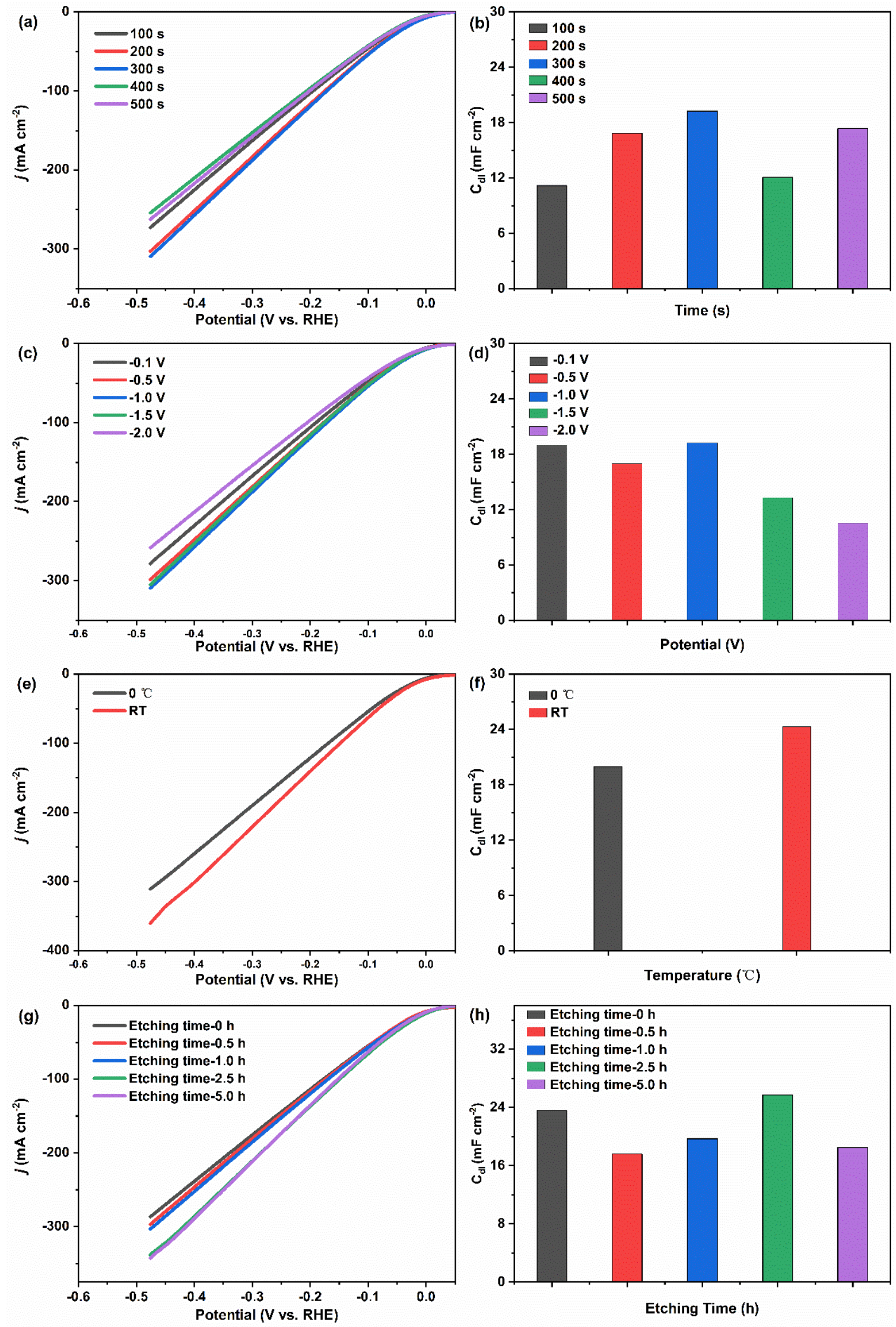

Figure S15. The HER LSVs curves and $\mathrm{C}_{\mathrm{d} l}$ were measured with different electrodeposition of $(\mathrm{a}, \mathrm{b})$ time, $(c, d)$ potential, $(e, f)$ temperature, and $(g, h)$ basic-etching time. 

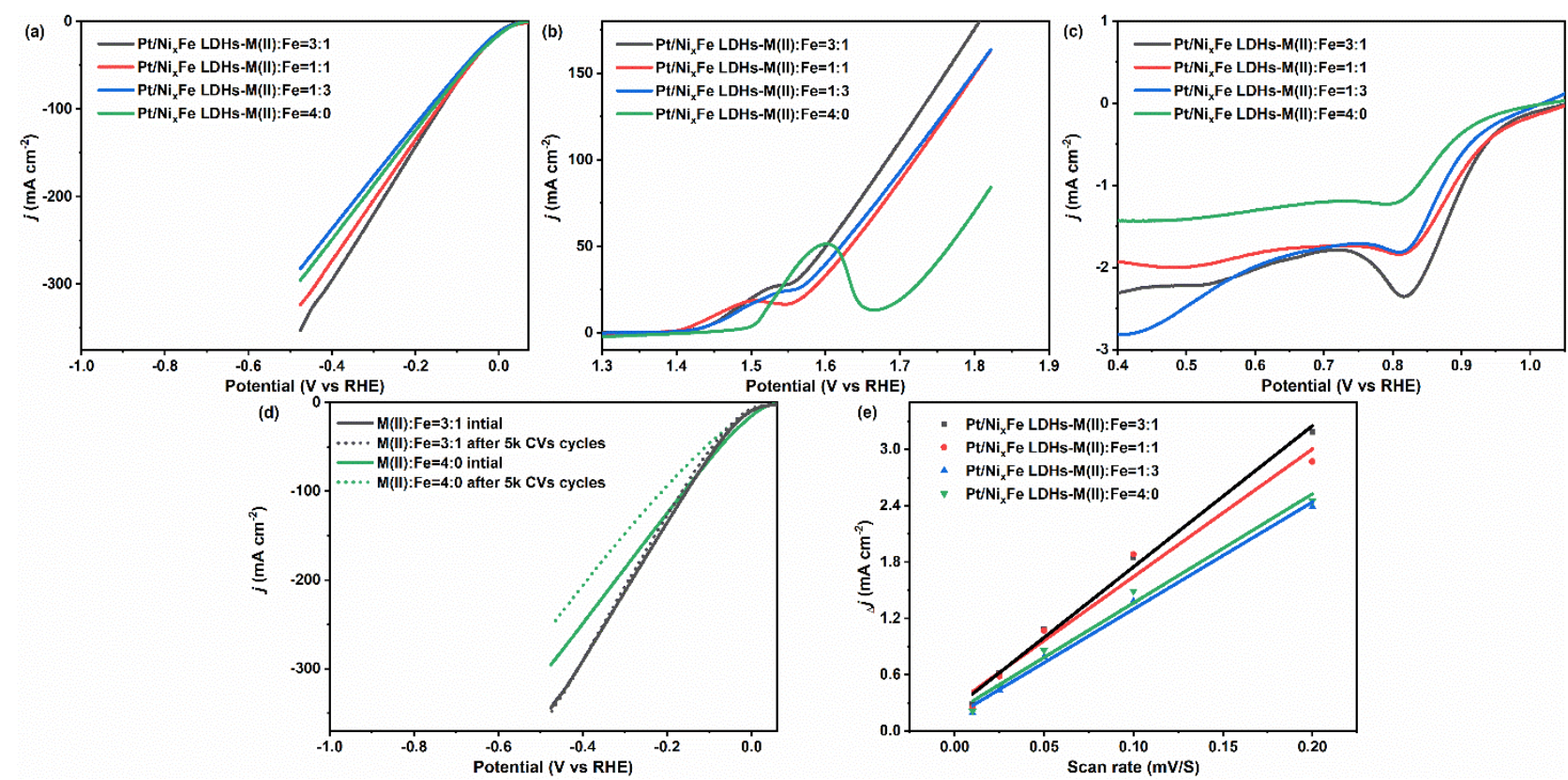

Figure S16. (a) HER, (b) OER and (c) ORR polarization curves, (d) HER stability and (e) $\mathrm{C}_{\mathrm{dl}}$ calculations of $\mathrm{Pt} / \mathrm{Ni}_{\mathrm{x}} \mathrm{Fe}$ LDHs with different proportions of Fe precursors: $\mathrm{M}(\mathrm{II}): \mathrm{Fe}^{3+}=3: 1,1: 1,1: 3$ and 4:0 $(\mathrm{M}=\mathrm{Ni}, \mathrm{Zn})$; Ni:Zn molar ratio was kept at 9:1.
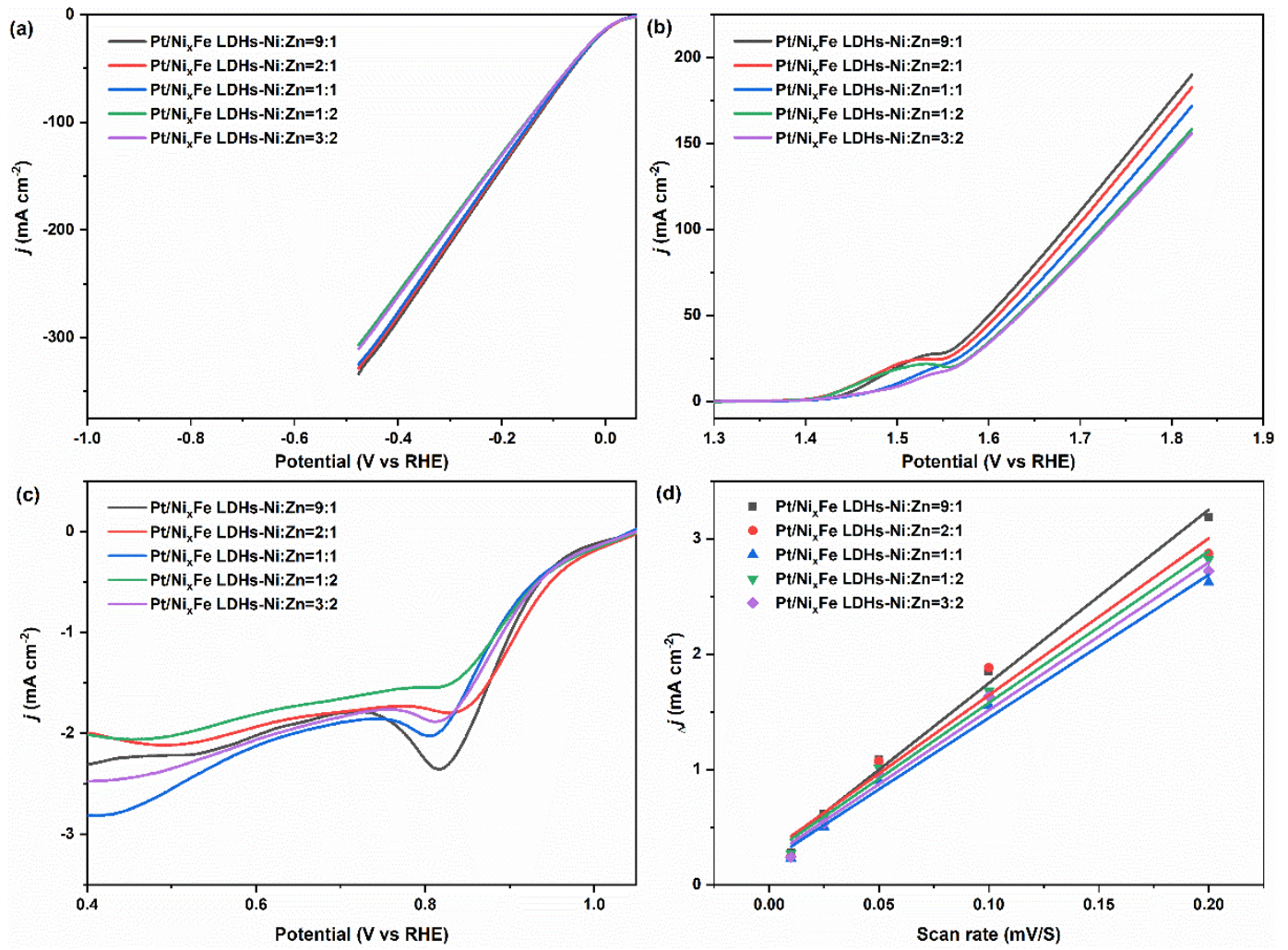

Figure S17. (a) HER, (b) OER and (c) ORR polarization curves and (d) $\mathrm{C} d$ calculations of $\mathrm{Pt} / \mathrm{Ni} \mathrm{x}_{\mathrm{Fe}}$ LDHs with different proportions of $\mathrm{Ni}$ and Zn precursors: $\mathrm{Ni}: \mathrm{Zn}=9: 1,2: 1,1: 1,2: 1$, and 3:2; $\mathrm{M}$ (II) : $\mathrm{Fe}^{3+}$ molar ratios was kept at 3:1 $(\mathrm{M}=\mathrm{Ni}, \mathrm{Zn})$. 

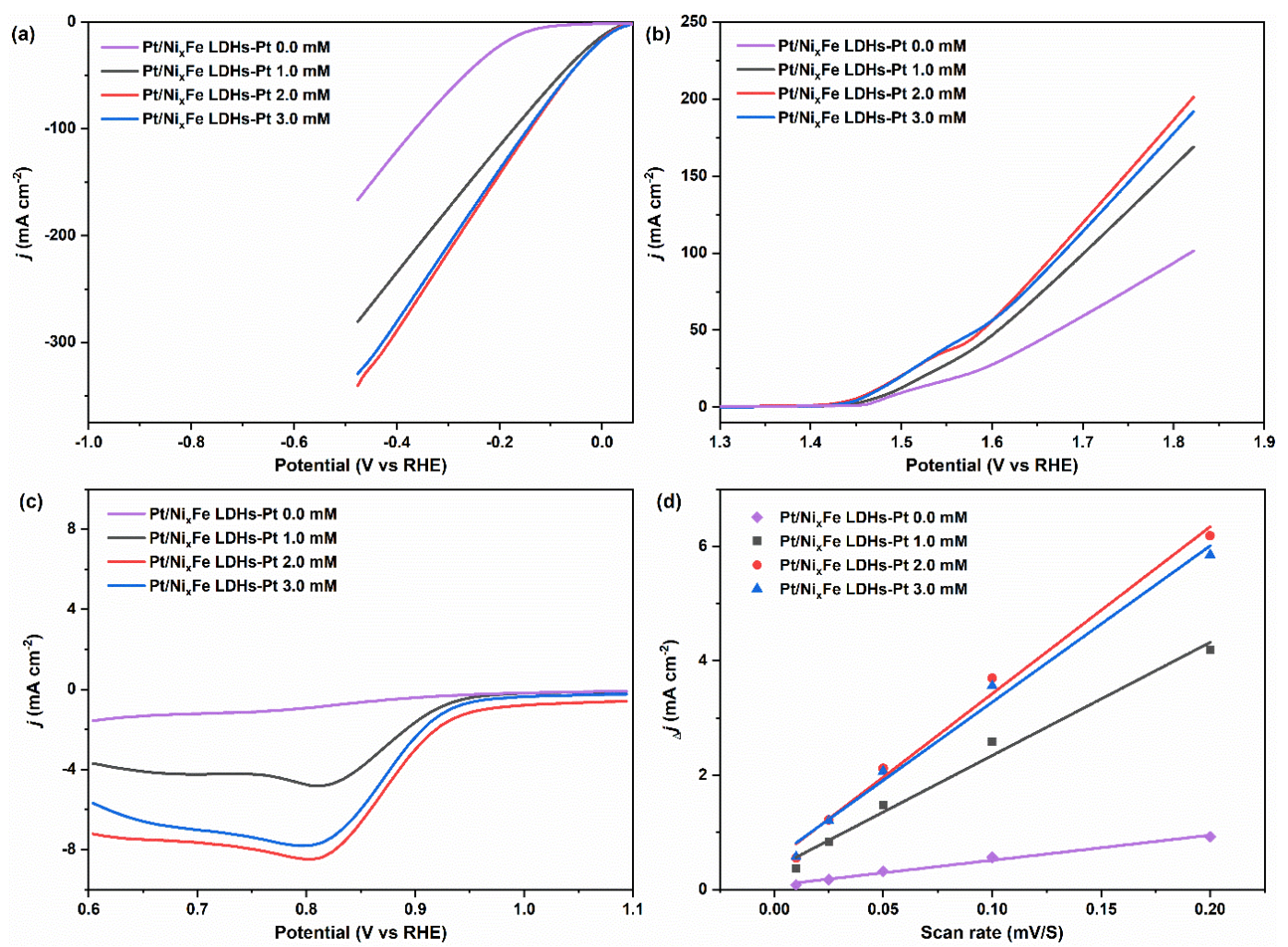

Figure S18. (a) HER, (b) OER and (c) ORR polarization curves and (d) $\mathrm{C}_{\mathrm{dl}}$ calculations of $\mathrm{Pt} / \mathrm{Ni} \mathrm{x}_{\mathrm{x}} \mathrm{Fe}$ LDHs with different concentration of Pt precursors: 0.0, 1.0, 2.0, and $3.0 \mathrm{mM} ; \mathrm{M}$ (II) : $\mathrm{Fe}^{3+}$ molar ratio was kept at 3:1 (M = Ni, Zn, Ni/Zn molar ratios was 9:1).
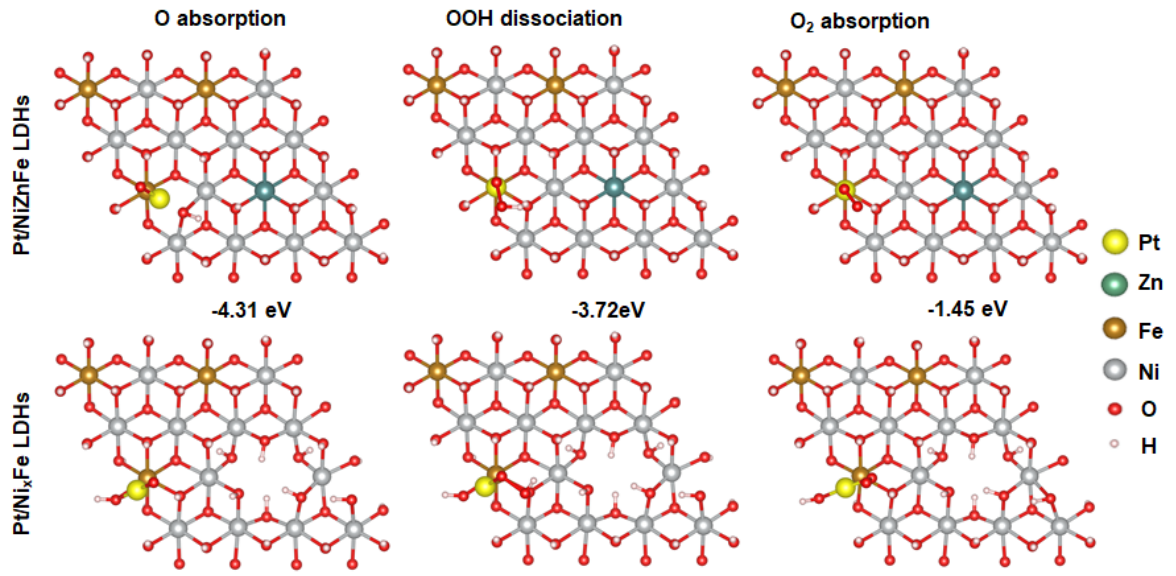

$-3.72 \mathrm{eV}$

$-1.45 \mathrm{eV}$
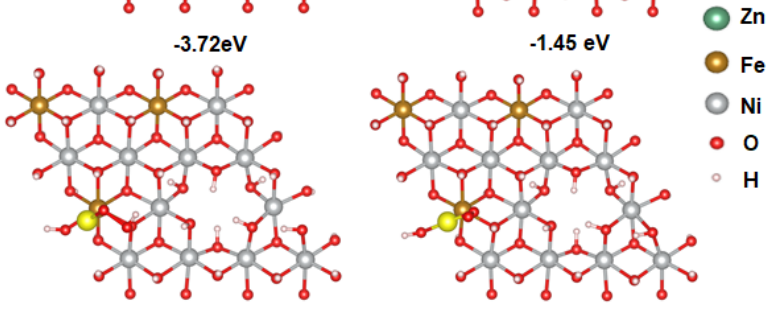

$-3.70 \mathrm{eV}$

$-3.62 \mathrm{eV}$

Figure S19. Calculated adsorption energies of $* \mathrm{O},{ }^{*} \mathrm{OOH}$, and $\mathrm{O}_{2}$ on $\mathrm{Pt} / \mathrm{Ni}_{x} \mathrm{Fe}$ LDHs and $\mathrm{Pt} / \mathrm{NiZnFe}$ LDHs surfaces. 


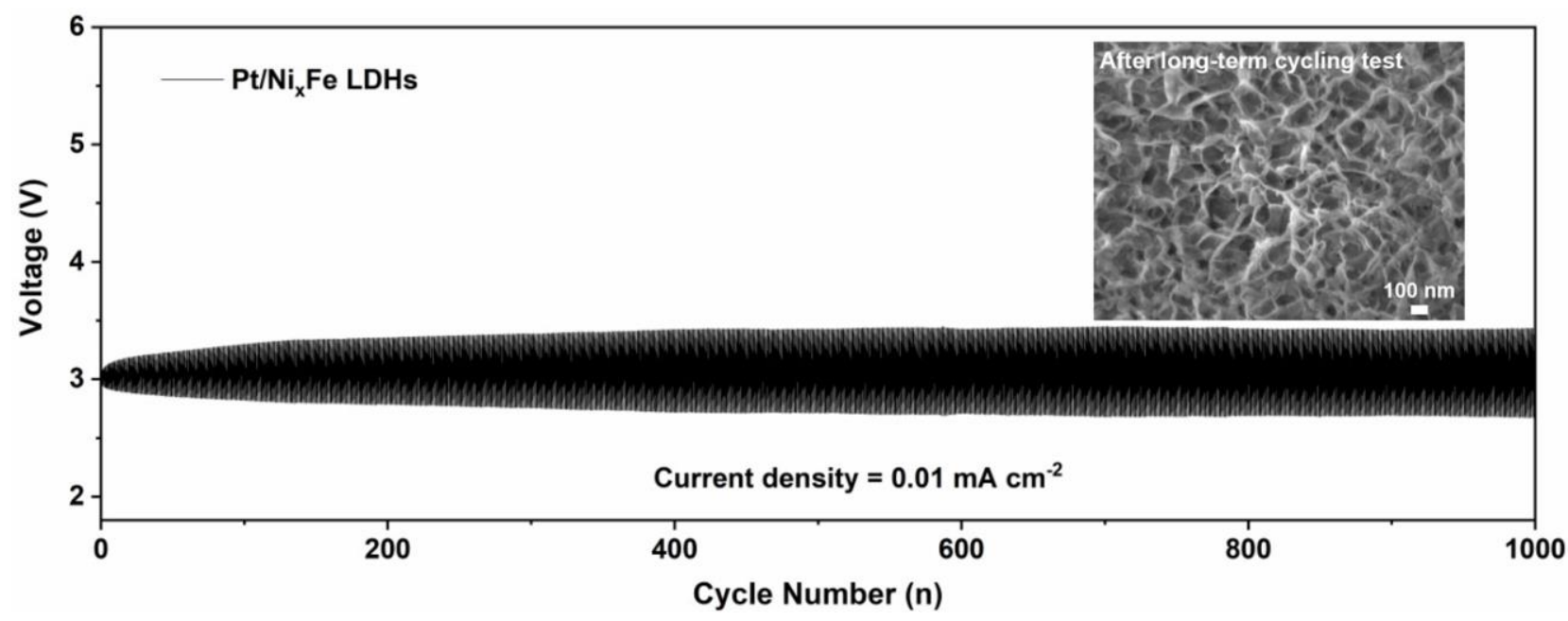

Figure S20. Cycling performance of hybrid sodium air battery using $\mathrm{Pt} / \mathrm{Ni}_{\mathrm{x}} \mathrm{Fe}$ LDHs air cathode at a current density of $0.01 \mathrm{~mA} \mathrm{~cm}^{-2}$ for 1000 cycles. Inset is the SEM image of $\mathrm{Pt} / \mathrm{NixFe} \mathrm{LDHs} \mathrm{cathode}$ after long term cycling test. 
Table S1. ICP-MS results of various catalysts.

\begin{tabular}{|c|c|c|c|c|c|}
\hline Sample & $\begin{array}{c}\text { Pt content } \\
(\mathrm{mg} / \mathrm{L})\end{array}$ & $\begin{array}{l}\text { Average } \\
(\mathrm{mg} / \mathrm{L})\end{array}$ & $\begin{array}{c}\text { Relative Standard } \\
\text { Deviation (RSD, \%) }\end{array}$ & $\begin{array}{c}\text { Standard } \\
\text { Deviation (SD) }\end{array}$ & $\begin{array}{c}\text { Weight Ratio } \\
(\mathrm{wt} \%)\end{array}$ \\
\hline \multirow{3}{*}{$\mathrm{Pt} / \mathrm{Ni}_{\mathrm{x}} \mathrm{Fe}$ LDHs-Pt $1 \mathrm{mM}$} & 1.50 & \multirow{3}{*}{1.79} & \multirow{3}{*}{17.96} & \multirow{3}{*}{0.32} & \multirow{3}{*}{1.27} \\
\hline & 2.14 & & & & \\
\hline & 1.72 & & & & \\
\hline \multirow{3}{*}{$\mathrm{Pt} / \mathrm{Ni}_{\mathrm{x}} \mathrm{Fe}$ LDHs-Pt $2 \mathrm{mM}$} & 2.69 & \multirow{3}{*}{3.01} & \multirow{3}{*}{9.52} & \multirow{3}{*}{0.29} & \multirow{3}{*}{2.24} \\
\hline & 3.24 & & & & \\
\hline & 3.10 & & & & \\
\hline \multirow{3}{*}{$\mathrm{Pt} / \mathrm{Ni}_{\mathrm{x}} \mathrm{Fe}$ LDHs-Pt $3 \mathrm{mM}$} & 5.79 & \multirow{3}{*}{5.36} & \multirow{3}{*}{7.95} & \multirow{3}{*}{0.43} & \multirow{3}{*}{3.85} \\
\hline & 5.35 & & & & \\
\hline & 4.94 & & & & \\
\hline
\end{tabular}

Table S2. XPS quantitative analysis of various catalysts (at $\%)$.

\begin{tabular}{ccccccc}
\hline Samples & Ni 2p & Fe 2p & O 1s & C 1s & Pt 4f & Zn 2p \\
\hline NiFe LDHs & 12.31 & 4.11 & 55.56 & 28.02 & - & - \\
Pt/Ni ${ }_{x} F e$ LDHs & 9.93 & 3.94 & 38.2 & 46.9 & 0.38 & 0.65 \\
\hline
\end{tabular}


Table S3. Comparison of HER and OER performance in $1 \mathrm{M} \mathrm{KOH}$ of recently reported Pt or NiFe LDH-based electrocatalysts.

\begin{tabular}{|c|c|c|c|c|c|}
\hline \multirow{2}{*}{ Catalysts } & \multirow[b]{2}{*}{$\eta_{10}(\mathrm{mV})$} & \multicolumn{2}{|l|}{ HER } & OER & \multirow{2}{*}{ Reference } \\
\hline & & Tafel slope $\left(\mathrm{mV} \mathrm{dec}{ }^{-1}\right)$ & $\eta_{10}(\mathrm{mV})$ & Tafel slope $\left(\mathrm{mV} \mathrm{dec}^{-1}\right)$ & \\
\hline $\mathrm{Pt} / \mathrm{Ni}_{\mathrm{x}} \mathrm{Fe} \mathrm{LDHs}$ & 5 & 81.7 & 186 & 36.3 & This work \\
\hline $\mathrm{Pt} / \mathrm{NiFe} \mathrm{LDHs}$ & 7 & 95.7 & 190 & 53.4 & This work \\
\hline $\mathrm{Ni}_{\mathrm{x}} \mathrm{Fe}$ LDHs & 152 & 132.3 & 221 & 87.9 & This work \\
\hline $\mathrm{NiFe}$ LDHs & 187 & 145.7 & 283 & 187.9 & This work \\
\hline $20 \% \mathrm{Pt} / \mathrm{C}$ & 73 & 207.6 & - & - & This work \\
\hline $20 \% \mathrm{Ir} / \mathrm{C}$ & - & - & 348 & 93.5 & This work \\
\hline $\mathrm{RuO}_{2}$ & - & - & 415 & 115.4 & This work \\
\hline $\mathrm{Ni}$ foam & 216 & 133.1 & 584 & 229.7 & This work \\
\hline $\mathrm{NiCo}_{2} \mathrm{~S}_{4} @ \mathrm{NiFe} \mathrm{LDH}$ & 200 & 48 & $201\left(\eta_{60}\right)$ & 46.3 & 3 \\
\hline $\mathrm{CoFe} @ \mathrm{NiFe}-200 / \mathrm{NF}$ & 240 & 84.69 & 190 & 45.71 & 4 \\
\hline DSO NiFe LDH & 59 & 62.3 & 184 & 28.83 & 5 \\
\hline Ru-doped NiFe LDH & 29 & 31 & 225 & - & 6 \\
\hline Pd-NiFe LDH & 130 & 46 & 156 & 58 & 7 \\
\hline $\mathrm{Pt}_{2} \mathrm{FeNi} / \mathrm{NiFe} \mathrm{LDH}$ & - & - & 179 & 90.3 & 8 \\
\hline Ag@NiFe LDHs & 200 & 87 & 330 & 89 & 9 \\
\hline (2D)-Pt on NiFe-LDH & 32.3 & 31 & - & - & 10 \\
\hline $\mathrm{Pt} / \mathrm{NiRu}-\mathrm{OH}$ & 38 & 37 & - & - & 11 \\
\hline Pt-NiFe-LDH-0.5-12 & $86(\eta 50)$ & 46 & $314\left(\eta_{50}\right)$ & 64 & 12 \\
\hline $\mathrm{Pt} / \mathrm{Co}-\mathrm{LDH}$ & - & - & 265 & 63 & 13 \\
\hline Pt-NiFe LDH/CC & 28 & 39 & - & - & 14 \\
\hline NiFeIr LDH & 34 & 32 & - & - & 15 \\
\hline Cu@NiFe LDH & 116 & 58.9 & 199 & 27.8 & 16 \\
\hline Ni-Fe LDH-NS@DG10 & 300 & 110 & 210 & 52 & 17 \\
\hline
\end{tabular}


Table S4. Electrolyte resistance $\left(\mathrm{R}_{\mathrm{s}}\right)$ and Charge-transfer resistance $\left(\mathrm{R}_{\mathrm{ct}}\right)$ values of various catalysts fitted in $1 \mathrm{M} \mathrm{KOH}$ solution.

\begin{tabular}{ccccc}
\hline \multirow{2}{*}{ Catalysts } & \multicolumn{2}{c}{ HER $(\eta=100 \mathrm{mV})$} & \multicolumn{2}{c}{ OER $(\eta=300 \mathrm{mV})$} \\
& $\mathrm{R}_{\mathrm{s}}(\Omega)$ & $\mathrm{R}_{\text {ct }}(\Omega)$ & $\mathrm{R}_{\mathrm{s}}(\Omega)$ & $\mathrm{R}_{\text {ct }}(\Omega)$ \\
\hline Ni foam & 1.28 & 74.80 & 1.30 & 9.82 \\
NiFe LDHs & 1.33 & 38.03 & 1.30 & 3.36 \\
NixFe LDHs & 1.30 & 17.93 & 1.30 & 1.63 \\
$\mathrm{Pt} / \mathrm{NiFe} \mathrm{LDHs}$ & 1.32 & 0.63 & 1.33 & 0.92 \\
$\mathrm{Pt} / \mathrm{Ni}$ Fe LDHs & 1.09 & 0.46 & 1.08 & 0.64 \\
\hline
\end{tabular}

Table S5. Comparison of overall water splitting performance of recently reported NiFe LDH-based bifunctional electrocatalysts.

\begin{tabular}{|c|c|c|}
\hline Catalysts & $\mathrm{E}_{j=10}(\mathrm{~V})$ & References \\
\hline $\mathrm{Pt} / \mathrm{Ni}_{x} \mathrm{Fe} \mathrm{LDHs}$ & 1.47 & This work \\
\hline $20 \% \mathrm{Ir} / \mathrm{C} \| 20 \% \mathrm{Pt} / \mathrm{C}$ & 1.59 & This work \\
\hline $\mathrm{RuO}_{2} \| 20 \% \mathrm{Pt} / \mathrm{C}$ & 1.62 & This work \\
\hline P-V-NiFe LDH NSA & 1.43 & 1 \\
\hline Pt-NiFe-LDH-0.5-12 & $1.63($ oER $j=50)$ & 12 \\
\hline NiFe LDH@NiCoP/NF & 1.57 & 18 \\
\hline CoFe-LDH@NiFe-LDH/NF & 1.59 & 4 \\
\hline DSO NiFe LDH NSA & 1.48 & 5 \\
\hline $\mathrm{Cu} @ \mathrm{CoFe}-\mathrm{LDH}$ & 1.681 & 19 \\
\hline NiFe LDH@NiCoP & 1.57 & 18 \\
\hline $\mathrm{Ru}-\mathrm{NiFe}-\mathrm{P}$ & 1.47 & 20 \\
\hline $\mathrm{NiFe} \mathrm{LDH} / \mathrm{NiCo}_{2} \mathrm{O}_{4} / \mathrm{NF}$ & 1.60 & 21 \\
\hline $\mathrm{Pt}-\mathrm{NiFe} \mathrm{LDH} / \mathrm{CC}$ & 1.52 & 14 \\
\hline Cu@NiFe LDH & 1.54 & 16 \\
\hline $\mathrm{NiCo}_{2} \mathrm{~S}_{4} @ \mathrm{NiFe} \mathrm{LDH}$ & 1.60 & 3 \\
\hline Ag NWs@ $@ \mathrm{Ni}_{0.95} \mathrm{Fe}_{0.05}$ LDHs & 1.70 & 9 \\
\hline
\end{tabular}


Table S6. ORR and OER bifunctional performance in $0.1 \mathrm{M} \mathrm{KOH}$ of recently reported NiFe LDHbased electrocatalysts.

\begin{tabular}{|c|c|c|c|c|}
\hline Catalysts & $E_{j}=10$, OER $(V)$ & $\mathrm{E}_{1 / 2, \mathrm{ORR}}(\mathrm{V})$ & $\triangle \mathrm{E}(\mathrm{V})$ & References \\
\hline $\mathrm{Pt} / \mathrm{Ni}_{\mathrm{x}} \mathrm{Fe} \mathrm{LDHs}$ & 1.59 & 0.87 & 0.72 & This work \\
\hline Pt-NiFe-LDH-0.5-12 & 1.55 & 0.84 & 0.71 & 12 \\
\hline nNiFe LDH/3D MPC & 1.54 & 0.83 & 0.71 & 22 \\
\hline Co-NC@NiFe LDH & 1.62 & 0.8 & 0.82 & 23 \\
\hline $\mathrm{NiCo}_{2} \mathrm{~S}_{4} @ \mathrm{NiFe} \mathrm{LDH}$ & 1.52 & 0.85 & 0.67 & 24 \\
\hline NiFe-LDH/rGO & 1.49 & 0.75 & 0.74 & 25 \\
\hline $\mathrm{NiFe}-\mathrm{LDH}$ & 1.61 & 0.5 & 1.11 & 26 \\
\hline $\mathrm{NiFe}-\mathrm{LDH} / \mathrm{Co}, \mathrm{N}-\mathrm{CNF}$ & 1.54 & 0.79 & 0.75 & 27 \\
\hline O-NiCoFe-LDH & 1.68 & 0.63 & 1.05 & 28 \\
\hline $\mathrm{Fe}-\mathrm{N}-\mathrm{C} / \mathrm{NiFe} \mathrm{LDH}$ & 1.54 & 0.79 & 0.75 & 29 \\
\hline $\mathrm{NiCo}^{\mathrm{III}} \mathrm{Fe}-\mathrm{LDH} / \mathrm{N}-\mathrm{GO}$ & 1.55 & $0.78(j=-3)$ & 0.77 & 30 \\
\hline $\mathrm{Co}_{3} \mathrm{O}_{4} @ \mathrm{NiFe} \mathrm{LDH}$ & - & - & 0.79 & 31 \\
\hline
\end{tabular}


Table S7. Performances of recently reported hybrid Na-air battery.

\begin{tabular}{|c|c|c|c|c|c|c|}
\hline Cathode & $\begin{array}{l}\text { Open-circuit } \\
\text { voltage }(\mathrm{V})\end{array}$ & $\begin{array}{c}\text { Charge/Discharge } \\
\text { voltage }(\mathrm{V})\end{array}$ & $\begin{array}{l}\text { Voltage } \\
\text { gap (V) }\end{array}$ & $\begin{array}{c}\text { Roundtrip } \\
\text { efficiency (\%) }\end{array}$ & Durability & Reference \\
\hline $\mathrm{Pt} / \mathrm{Ni}_{\mathrm{x}} \mathrm{Fe} \mathrm{LDHs}$ & 3.01 & $3.34 / 2.93$ & 0.41 & 87.7 & $\geq 1000$ cycles $/ 0.01 \mathrm{~mA} \mathrm{~cm}^{-2}$ & This work \\
\hline $20 \% \mathrm{Pt} / \mathrm{C}+20 \% \mathrm{Ir} / \mathrm{C}$ & 2.95 & $3.29 / 2.91$ & 0.38 & 88.4 & $<30$ cycles $/ 0.01 \mathrm{~mA} \mathrm{~cm}{ }^{-2}$ & This work \\
\hline $\mathrm{RuO}_{2}$ & 2.83 & $3.41 / 2.80$ & 0.61 & 82.1 & $<30$ cycles $/ 0.01 \mathrm{~mA} \mathrm{~cm}^{-2}$ & This work \\
\hline MOF-NCNTs & - & $3.10 / 2.80$ & 0.30 & 90 & 35 cycles $/ 0.01 \mathrm{~mA} \mathrm{~cm}^{-2}$ & 32 \\
\hline Co-N-C-0.5 & 3.10 & $3.11 / 2.80$ & 0.38 & 90.03 & 20 cycles $/ 0.1 \mathrm{~mA} \mathrm{~cm}^{-2}$ & 33 \\
\hline $\mathrm{Bi}_{2} \mathrm{Ru}_{2} \mathrm{O}_{7}$ & $\sim 3.17$ & $/ \sim 3.17$ & 0.211 & 93.58 & 50 cycles $/ 0.01 \mathrm{~mA} \mathrm{~cm}^{-2}$ & 34 \\
\hline VGC & 3.06 & $3.45 / 2.81$ & 0.64 & 81 & 50 cycles $/ 4 \mathrm{~mA} \mathrm{~g}^{-1}$ & 35 \\
\hline $\mathrm{P}-\mathrm{Tl}_{2} \mathrm{Ru}_{2} \mathrm{O}_{7}$ & $\begin{array}{c}3.17 \\
\text { (theoretical) }\end{array}$ & $3.23 / 3.07$ & 0.16 & 95 & 50 cycles $/ 0.01 \mathrm{~mA} \mathrm{~cm}^{-2}$ & 36 \\
\hline CuS-CNT & - & - & - & $90 \%$ & 100 cycles $/ 0.5 \mathrm{~mA} \mathrm{~cm}^{-2}$ & 37 \\
\hline Ni nanoparticles & - & $3.22 / 2.65$ & 0.57 & 80.3 & 100 cycles $/ 0.1 \mathrm{~mA} \mathrm{~cm}^{-2}$ & 38 \\
\hline $\mathrm{SnS}_{2}$ & 2.8 & $3.18 / 2.66$ & 0.52 & $83 \%$ & 40 cycles $/ 5 \mathrm{~mA} \mathrm{~g}^{-1}$ & 39 \\
\hline $\mathrm{Co}_{3}\left(\mathrm{PO}_{4}\right)_{2}$ & $\begin{array}{c}3.48 \\
\text { (theoretical) }\end{array}$ & $3.41 / 2.82$ & 0.59 & 83 & 50 cycles $/ 0.05 \mathrm{~mA} \mathrm{~cm}^{-2}$ & 40 \\
\hline $\begin{array}{c}\mathrm{MnO}_{2} / \mathrm{rGO} / \text { carbon } \\
\text { paper }\end{array}$ & $\begin{array}{c}3.1 \\
\text { (theoretical) }\end{array}$ & $3.59 / 2.9$ & 0.7 & 81 & 20 cycles $/ 15 \mathrm{~mA} \mathrm{~g}^{-1}$ & 41 \\
\hline S-rGO-CNT-Co & $\begin{array}{c}3.48 \\
\text { (theoretical) }\end{array}$ & $3.42 / 3.00$ & 0.42 & 87.8 & 50 cycles $/ 0.01 \mathrm{~mA} \mathrm{~cm}^{-2}$ & 42 \\
\hline $\mathrm{CMO}$ & $\begin{array}{c}3.48 \\
\text { (theoretical) }\end{array}$ & $3.71 / 3.13$ & 0.58 & 84.4 & 30 cycles $/ 0.01 \mathrm{~mA} \mathrm{~cm}^{-2}$ & 43 \\
\hline $\mathrm{dp}-\mathrm{MnCo}_{2} \mathrm{O}_{4} / \mathrm{N}-\mathrm{rGO}$ & - & $3.14 / 2.75$ & 0.39 & 87.6 & 25 cycles $/ 0.13 \mathrm{~mA} \mathrm{~cm}^{-2}$ & 44 \\
\hline
\end{tabular}




\section{References}

(1) Tang, Y.; Liu, Q.; Dong, L.; Wu, H. B.; Yu, X.-Y. Activating the Hydrogen Evolution and Overall Water Splitting Performance of NiFe LDH by Cation Doping and Plasma Reduction. Appl. Catal. B 2020, 266, 118627.

(2) Lv, J.; Liu, P.; Yang, F.; Xing, L.; Wang, D.; Chen, X.; Gao, H.; Huang, X.; Lu, Y.; Wang, G. 3d Hydrangea Macrophylla-Like Nickel-Vanadium Metal-Organic Frameworks Formed by SelfAssembly of Ultrathin 2D Nanosheets for Overall Water Splitting. ACS Appl. Mater. Interfaces 2020, $12,48495-48510$.

(3) Liu, J.; Wang, J.; Zhang, B.; Ruan, Y.; Lv, L.; Ji, X.; Xu, K.; Miao, L.; Jiang, J. Hierarchical NiCo2S4@NiFe LDH Heterostructures Supported on Nickel Foam for Enhanced Overall-WaterSplitting Activity. ACS Appl. Mater. Interfaces 2017, 9, 15364-15372.

(4) Yang, R.; Zhou, Y.; Xing, Y.; Li, D.; Jiang, D.; Chen, M.; Shi, W.; Yuan, S. Synergistic Coupling of CoFe-LDH Arrays with NiFe-LDH Nanosheet for Highly Efficient Overall Water Splitting in Alkaline Media. Appl. Catal. B 2019, 253, 131-139.

(5) Qiu, Z.; Tai, C.-W.; Niklasson, G. A.; Edvinsson, T. Direct Observation of Active Catalyst Surface Phases and the Effect of Dynamic Self-Optimization in NiFe-Layered Double Hydroxides for Alkaline Water Splitting. Energy Environ. Sci. 2019, 12, 572-581.

(6) Chen, G.; Wang, T.; Zhang, J.; Liu, P.; Sun, H.; Zhuang, X.; Chen, M.; Feng, X. Accelerated Hydrogen Evolution Kinetics on NiFe-Layered Double Hydroxide Electrocatalysts by Tailoring Water Dissociation Active Sites. Adv. Mater. 2018, 30, 1706279.

(7) Chen, R.; Hung, S. F.; Zhou, D.; Gao, J.; Yang, C.; Tao, H.; Yang, H. B.; Zhang, L.; Zhang, L.; Xiong, Q. Layered Structure Causes Bulk NiFe Layered Double Hydroxide Unstable in Alkaline Oxygen Evolution Reaction. Adv. Mater. 2019, 31, 1903909.

(8) Zheng, Z.; Guo, Y.; Wan, H.; Chen, G.; Zhang, N.; Ma, W.; Liu, X.; Liang, S.; Ma, R. Anchoring Active Sites by $\mathrm{Pt}_{2} \mathrm{FeNi}$ Alloy Nanoparticles on NiFe Layered Double Hydroxides for Efficient Electrocatalytic Oxygen Evolution Reaction. Energy Environ. Mater. 2021, 0, 1-8.

(9) Zhang, X.; Marianov, A. N.; Jiang, Y.; Cazorla, C.; Chu, D. Hierarchically Constructed Silver Nanowire@Nickel-Iron Layered Double Hydroxide Nanostructures for Electrocatalytic Water Splitting. Appl. Nano Mater. 2019, 3, 887-895.

(10) Jang, S. W.; Dutta, S.; Kumar, A.; Hong, Y.-R.; Kang, H.; Lee, S.; Ryu, S.; Choi, W.; Lee, I. S. Holey Pt Nanosheets on NiFe-Hydroxide Laminates: Synergistically Enhanced Electrocatalytic 2D Interface toward Hydrogen Evolution Reaction. ACS Nano 2020, 14, 10578-10588.

(11) Li, D.; Chen, X.; Lv, Y.; Zhang, G.; Huang, Y.; Liu, W.; Li, Y.; Chen, R.; Nuckolls, C.; Ni, H. An Effective Hybrid Electrocatalyst for the Alkaline HER: Highly Dispersed Pt Sites Immobilized by a Functionalized Niru-Hydroxide. Appl. Catal. B 2020, 269, 118824.

(12) Han, J.; Meng, X.; Lu, L.; Wang, Z. L.; Sun, C. Triboelectric Nanogenerators Powered Electrodepositing Tri-Functional Electrocatalysts for Water Splitting and Rechargeable Zinc-Air Battery: A Case of Pt Nanoclusters on NiFe-LDH Nanosheets. Nano Energy 2020, 104669.

(13) Zhang, S.; Zhan, G.; Wang, X.; Cao, S.; Yang, Q.; Yang, L.; Li, M.; Han, J.; Zhu, X.; Wang, 
H. Well-Defined Co-Pt-OH as "Electronic Pump" on Co-LDH Nanocages for Enhanced Oxygen Evolution Reaction. Appl. Catal. B 2020, 269, 118782.

(14) Yan, Q.; Yan, P.; Wei, T.; Wang, G.; Cheng, K.; Ye, K.; Zhu, K.; Yan, J.; Cao, D.; Li, Y. A Highly Efficient and Durable Water Splitting System: Platinum Sub-Nanocluster Functionalized Nickel-Iron Layered Double Hydroxide as the Cathode and Hierarchical Nickel-Iron Selenide as the Anode. J. Mater. Chem. A 2019, 7, 2831-2837.

(15) Chen, Q.-Q.; Hou, C.-C.; Wang, C.-J.; Yang, X.; Shi, R.; Chen, Y. Ir ${ }^{4+}$-Doped NiFe LDH to Expedite Hydrogen Evolution Kinetics as a Pt-Like Electrocatalyst for Water Splitting. Chem. Commun. 2018, 54, 6400-6403.

(16) Yu, L.; Zhou, H.; Sun, J.; Qin, F.; Yu, F.; Bao, J.; Yu, Y.; Chen, S.; Ren, Z. Cu Nanowires Shelled with NiFe Layered Double Hydroxide Nanosheets as Bifunctional Electrocatalysts for Overall Water Splitting. Energy Environ. Sci. 2017, 10, 1820-1827.

(17) Jia, Y.; Zhang, L.; Gao, G.; Chen, H.; Wang, B.; Zhou, J.; Soo, M. T.; Hong, M.; Yan, X.; Qian, G. A Heterostructure Coupling of Exfoliated Ni-Fe Hydroxide Nanosheet and Defective Graphene as a Bifunctional Electrocatalyst for Overall Water Splitting. Adv. Mater. 2017, 29, 1700017.

(18) Zhang, H.; Li, X.; Hähnel, A.; Naumann, V.; Lin, C.; Azimi, S.; Schweizer, S. L.; Maijenburg, A. W.; Wehrspohn, R. B. Bifunctional Heterostructure Assembly of NiFe LDH Nanosheets on NiCoP Nanowires for Highly Efficient and Stable Overall Water Splitting. Adv. Funct. Mater. 2018, 28, 1706847.

(19) Yu, L.; Zhou, H.; Sun, J.; Qin, F.; Luo, D.; Xie, L.; Yu, F.; Bao, J.; Li, Y.; Yu, Y. Hierarchical $\mathrm{Cu} @$ CoFe Layered Double Hydroxide Core-Shell Nanoarchitectures as Bifunctional Electrocatalysts for Efficient Overall Water Splitting. Nano Energy 2017, 41, 327-336.

(20) Qu, M.; Jiang, Y.; Yang, M.; Liu, S.; Guo, Q.; Shen, W.; Li, M.; He, R. Regulating Electron Density of NiFe-P Nanosheets Electrocatalysts by a Trifle of Ru for High-Efficient Overall Water Splitting. Appl. Catal. B 2020, 263, 118324.

(21) Wang, Z.; Zeng, S.; Liu, W.; Wang, X.; Li, Q.; Zhao, Z.; Geng, F. Coupling Molecularly Ultrathin Sheets of NiFe-Layered Double Hydroxide on $\mathrm{NiCo}_{2} \mathrm{O}_{4}$ Nanowire Arrays for Highly Efficient Overall Water-Splitting Activity. ACS Appl. Mater. Interfaces 2017, 9, 1488-1495.

(22) Wang, W.; Liu, Y.; Li, J.; Luo, J.; Fu, L.; Chen, S. NiFe LDH Nanodots Anchored on 3D Macro/Mesoporous Carbon as a High-Performance ORR/OER Bifunctional Electrocatalyst. J. Mater. Chem. A 2018, 6, 14299-14306.

(23) Chen, D.; Chen, X.; Cui, Z.; Li, G.; Han, B.; Zhang, Q.; Sui, J.; Dong, H.; Yu, J.; Yu, L. DualActive-Site Hierarchical Architecture Containing NiFe-LDH and ZIF-Derived Carbon-Based Framework Composite as Efficient Bifunctional Oxygen Electrocatalysts for Durable Rechargeable Zn-Air Batteries. Chem. Eng. J. 2020, 399, 125718.

(24) Feng, X.; Jiao, Q.; Chen, W.; Dang, Y.; Dai, Z.; Suib, S. L.; Zhang, J.; Zhao, Y.; Li, H.; Feng, C. Cactus-Like NiCo2 $\mathrm{S}_{4} @ \mathrm{NiFe}$ LDH Hollow Spheres as an Effective Oxygen Bifunctional Electrocatalyst in Alkaline Solution. Appl. Catal. B 2021, 286, 119869.

(25) Zhan, T.; Liu, X.; Lu, S.; Hou, W. Nitrogen Doped NiFe Layered Double Hydroxide/Reduced Graphene Oxide Mesoporous Nanosphere as an Effective Bifunctional Electrocatalyst for Oxygen 
Reduction and Evolution Reactions. Appl. Catal. B 2017, 205, 551-558.

(26) Zhang, M.; Zhang, J.; Ran, S.; Qiu, L.; Sun, W.; Yu, Y.; Chen, J.; Zhu, Z. A Robust Bifunctional Catalyst for Rechargeable Zn-Air Batteries: Ultrathin NiFe-LDH Nanowalls Vertically Anchored on Soybean-Derived Fe-NC Matrix. Nano Res. 2021, 14, 1175-1186.

(27) Wang, Q.; Shang, L.; Shi, R.; Zhang, X.; Zhao, Y.; Waterhouse, G. I.; Wu, L. Z.; Tung, C. H.; Zhang, T. NiFe Layered Double Hydroxide Nanoparticles on Co, N-Codoped Carbon Nanoframes as Efficient Bifunctional Catalysts for Rechargeable Zinc-Air Batteries. Adv. Energy Mater. 2017, 7 , 1700467.

(28) Qian, L.; Lu, Z.; Xu, T.; Wu, X.; Tian, Y.; Li, Y.; Huo, Z.; Sun, X.; Duan, X. Trinary Layered Double Hydroxides as High-Performance Bifunctional Materials for Oxygen Electrocatalysis. Adv. Energy Mater. 2015, 5, 1500245.

(29) Dresp, S.; Luo, F.; Schmack, R.; Kühl, S.; Gliech, M.; Strasser, P. An Efficient Bifunctional Two-Component Catalyst for Oxygen Reduction and Oxygen Evolution in Reversible Fuel Cells, Electrolyzers and Rechargeable Air Electrodes. Energy Environ. Sci. 2016, 9, 2020-2024.

(30) Zhou, D.; Cai, Z.; Lei, X.; Tian, W.; Bi, Y.; Jia, Y.; Han, N.; Gao, T.; Zhang, Q.; Kuang, Y. NiCoFe-Layered Double Hydroxides/N-Doped Graphene Oxide Array Colloid Composite as an Efficient Bifunctional Catalyst for Oxygen Electrocatalytic Reactions. Adv. Energy Mater. 2018, 8, 1701905 .

(31) Guo, X.; Hu, X.; Wu, D.; Jing, C.; Liu, W.; Ren, Z.; Zhao, Q.; Jiang, X.; Xu, C.; Zhang, Y. Tuning the Bifunctional Oxygen Electrocatalytic Properties of Core-Shell $\mathrm{Co}_{3} \mathrm{O}_{4} @ \mathrm{NiFe} \mathrm{LDH}$ Catalysts for Zn-Air Batteries: Effects of Interfacial Cation Valences. ACS Appl. Mater. Interfaces 2019, 11, 21506-21514.

(32) Wu, Y.; Qiu, X.; Liang, F.; Zhang, Q.; Koo, A.; Dai, Y.; Lei, Y.; Sun, X. A Metal-Organic Framework-Derived Bifunctional Catalyst for Hybrid Sodium-Air Batteries. Appl. Catal. B 2019, 241, 407-414.

(33) Zhu, J.; Qu, T.; Su, F.; Wu, Y.; Kang, Y.; Chen, K.; Yao, Y.; Ma, W.; Yang, B.; Dai, Y. Highly Dispersed Co Nanoparticles Decorated on a N-Doped Defective Carbon Nano-Framework for a Hybrid Na-Air Battery. Dalton Trans. 2020, 49, 1811-1821.

(34) Kim, M.; Ju, H.; Kim, J. Single Crystalline Bi2ru2o7 Pyrochlore Oxide Nanoparticles as Efficient Bifunctional Oxygen Electrocatalyst for Hybrid Na-Air Batteries. Chem. Eng. J. 2019, 358, $11-19$.

(35) Khan, Z.; Senthilkumar, B.; Park, S. O.; Park, S.; Yang, J.; Lee, J. H.; Song, H.-K.; Kim, Y.; Kwak, S. K.; Ko, H. Carambola-Shaped $\mathrm{VO}_{2}$ Nanostructures: A Binder-Free Air Electrode for an Aqueous Na-Air Battery. J. Mater. Chem. A 2017, 5, 2037-2044.

(36) Kim, M.; Ju, H.; Kim, J. Dihydrogen Phosphate Ion Functionalized Nanocrystalline Thallium Ruthenium Oxide Pyrochlore as a Bifunctional Electrocatalyst for Aqueous Na-Air Batteries. Appl. Catal. B 2019, 245, 29-39.

(37) Gross, M. M.; Manthiram, A. Development of Low-Cost Sodium-Aqueous Polysulfide Hybrid Batteries. Energy Storage Mater. 2019, 19, 346-351.

(38) Su, F.; Qiu, X.; Liang, F.; Tanaka, M.; Qu, T.; Yao, Y.; Ma, W.; Yang, B.; Dai, Y.; Hayashi, K. 
Preparation of Nickel Nanoparticles by Direct Current Arc Discharge Method and Their Catalytic Application in Hybrid Na-Air Battery. Nanomaterials 2018, 8, 684.

(39) Khan, Z.; Parveen, N.; Ansari, S. A.; Senthilkumar, S.; Park, S.; Kim, Y.; Cho, M. H.; Ko, H. Three-Dimensional $\mathrm{SnS}_{2}$ Nanopetals for Hybrid Sodium-Air Batteries. Electrochim. Acta 2017, 257, 328-334.

(40) Senthilkumar, B.; Khan, Z.; Park, S.; Seo, I.; Ko, H.; Kim, Y. Exploration of Cobalt Phosphate as a Potential Catalyst for Rechargeable Aqueous Sodium-Air Battery. J. Power Sources 2016, 311 , 29-34.

(41) Khan, Z.; Park, S.; Hwang, S. M.; Yang, J.; Lee, Y.; Song, H.-K.; Kim, Y.; Ko, H. Hierarchical Urchin-Shaped $\mathrm{A}-\mathrm{MnO}_{2}$ on Graphene-Coated Carbon Microfibers: A Binder-Free Electrode for Rechargeable Aqueous Na-Air Battery. NPG Asia Mater. 2016, 8, e294-e294.

(42) Suh, D. H.; Park, S. K.; Nakhanivej, P.; Kim, Y.; Hwang, S. M.; Park, H. S. Hierarchically Structured Graphene-Carbon Nanotube-Cobalt Hybrid Electrocatalyst for Seawater Battery. J. Power Sources 2017, 372, 31-37.

(43) Abirami, M.; Hwang, S. M.; Yang, J.; Senthilkumar, S. T.; Kim, J.; Go, W.-S.; Senthilkumar, B.; Song, H.-K.; Kim, Y. A Metal-Organic Framework Derived Porous Cobalt Manganese Oxide Bifunctional Electrocatalyst for Hybrid Na-Air/Seawater Batteries. ACS Appl. Mater. Interfaces 2016, 8, 32778-32787.

(44) Kang, Y.; Zou, D.; Zhang, J.; Liang, F.; Hayashi, K.; Wang, H.; Xue, D.; Chen, K.; Adair, K. R.; Sun, X. Dual-Phase Spinel $\mathrm{MnCo}_{2} \mathrm{O}_{4}$ Nanocrystals with Nitrogen-Doped Reduced Graphene Oxide as Potential Catalyst for Hybrid Na-Air Batteries. Electrochim. Acta 2017, 244, 222-229. 